Research article

\title{
Genetic mapping of putative Chrna7 and Luzp2 neuronal transcriptional enhancers due to impact of a transgene-insertion and 6.8 Mb deletion in a mouse model of Prader-Willi and Angelman syndromes
}

\author{
Mihaela Stefan ${ }^{1,7}$, Kathryn C Claiborn ${ }^{1}$, Edyta Stasiek ${ }^{3}$, Jing-Hua Chai ${ }^{1}$, \\ Tohru Ohta ${ }^{1,6}$, Richard Longnecker ${ }^{4}$, John M Greally ${ }^{3}$ and \\ Robert D Nicholls*1,2,5,7
}

Address: ${ }^{1}$ Center for Neurobiology and Behavior, Department of Psychiatry, University of Pennsylvania, Philadelphia, PA 19104, USA, ${ }^{2}$ Department of Genetics, University of Pennsylvania, Philadelphia, PA 19104, USA, ${ }^{3}$ Division of Hematology, Department of Medicine, Albert Einstein College of Medicine, The Bronx, USA, ${ }^{4}$ Department of Microbiology and Immunology, Feinberg School of Medicine, Northwestern University, Ward 6-231, 303 East Chicago Ave, Chicago, IL 60611, USA, ${ }^{3}$ Birth Defects Laboratories, Children's Hospital of Pittsburgh, Room 2109 Rangos Research Center, 3460 Fifth Avenue, Pittsburgh, PA 15213, USA, ${ }^{6}$ Health Science University of Hokkaido, Hokkaido, Japan and ${ }^{7}$ Department of Pediatrics, Children's Hospital of Pittsburgh, 3460 Fifth Avenue, Pittsburgh, PA 15213

Email: Mihaela Stefan - Mihaela.Stefan@chp.edu; Kathryn C Claiborn - kcc2@mail.med.upenn.edu; Edyta Stasiek - estasiek@hotmail.com; JingHua Chai - jinghua@mail.med.upenn.edu; Tohru Ohta - ohta@hoku-iryo-u.ac.jp; Richard Longnecker - r-longnecker@northwestern.edu; John M Greally - jgreally@aecom.yu.edu; Robert D Nicholls* - Robert.Nicholls@chp.edu

* Corresponding author

Published: 09 November 2005

Received: 14 July 2005

BMC Genomics 2005, 6:157 doi:10.1/86/|47|-2/64-6-157

Accepted: 09 November 2005

This article is available from: http://www.biomedcentral.com//47/-2/64/6//57

(c) 2005 Stefan et al; licensee BioMed Central Ltd.

This is an Open Access article distributed under the terms of the Creative Commons Attribution License (http://creativecommons.org/licenses/by/2.0), which permits unrestricted use, distribution, and reproduction in any medium, provided the original work is properly cited.

\begin{abstract}
Background: Prader-Willi and Angelman syndrome (PWS and AS) patients typically have an $\sim 5 \mathrm{Mb}$ deletion of human chromosome 15 $\mathrm{qI}$ l-q/3, of opposite parental origin. A mouse model of PWS and AS has a transgenic insertion-deletion (TgPWS/TgAS) of chromosome 7B/C subsequent to paternal or maternal inheritance, respectively. In this study, we define the deletion endpoints and examine the impact on expression of flanking genes.

Results: Using molecular and cytological methods we demonstrate that 13 imprinted and II non-imprinted genes are included in the TgPWS/TgAS deletion. Normal expression levels were found in TgPWS brain for genes extending 9.1or 5.6-Mb centromeric or telomeric of the deletion, respectively. Our molecular cytological studies map the proximal deletion breakpoint between the Luzp2 and Siglec-H loci, and we show that overall mRNA levels of Luzp2 in TgPWS and TgAS brain are significantly reduced by 17\%. Intriguingly, 5' Chrna7 shows I.7-fold decreased levels in TgPWS and TgAS brain whereas there is a $\geq 15$-fold increase in expression in neonatal liver and spleen of these mouse models. By isolating a Chrna7-Tg fusion transcript from TgAS mice, we mapped the telomeric deletion breakpoint in Chrna7 intron 4.

Conclusion: Based on the extent of the deletion, TgPWS/TgAS mice are models for PWS/AS class I deletions. Other than for the first gene promoters immediately outside the deletion, since genes extending 5.6-9.1 Mb away from each end of the deletion show normal expression levels in TgPWS brain, this indicates that the transgene array does not induce silencing and there are no additional linked rearrangements. Using gene expression, non-coding conserved sequence (NCCS) and synteny data, we have genetically mapped a putative Luzp2 neuronal enhancer responsible for $\sim 33 \%$ of allelic transcriptional activity. The Chrna7 results are explained by hypothesizing loss of an essential neuronal transcriptional enhancer required for $\sim 80 \%$ of allelic Chrna7 promoter activity, while the Chrna7 promoter is upregulated in B lymphocytes by the transgene immunoglobulin enhancer. The mapping of a putative Chrna7 neuronal enhancer inside the deletion has significant implications for understanding the transcriptional regulation of this schizophrenia-susceptibility candidate gene.
\end{abstract}




\section{Background}

Prader-Willi and Angelman syndrome (PWS and AS) are complex neurobehavioral disorders associated with loss of function of a cluster of differentially expressed imprinted genes in chromosome 15q11-q13 [1]. PWS is characterized by a neonatal stage of failure to thrive, hypotonia and respiratory distress followed by hyperphagia in early childhood with development of severe obesity, as well as short stature, hypogonadism, small hands and feet, mild to moderate mental retardation, and obsessivecompulsive behavior $[2,3]$. In contrast, AS patients have a more pronounced neurological disease including developmental delay, severe mental retardation with lack of speech, hyperactivity, seizures, aggressive behavior and excessive inappropriate laughter [2]. Most PWS and AS cases ( $\sim 70 \%$ ) are due to $\sim 5 \mathrm{Mb}$ de novo deletions spanning a $2 \mathrm{Mb}$ imprinted domain and several adjacent nonimprinted genes [1]. There are two classes of deletions in PWS/AS patients, one from breakpoint 1 (BP1) to BP3 and the other from BP2 to BP3 [4]. Additionally, paternal or maternal uniparental disomy (pat or matUPD) explain $25 \%$ of PWS and $5 \%$ of AS cases, respectively, while $2-5 \%$ of PWS and AS cases result from imprinting defects (ID). In each mechanism, PWS arises from loss of ten paternally expressed loci, while AS arises from loss of function of the maternally expressed UBE3A gene [1].

Mouse models of PWS with either matUPD [5], an ID [6] or a paternally-inherited chromosome deletion [7] share a similar phenotype with failure to thrive, hypotonia and early postnatal lethality, modeling the first stage of the human syndrome $[9,10]$. Similarly, mouse models of AS have a patUPD [10], maternally-inherited chromosome deletion [7], or a maternal mutation of Ube3a [11,12]. In the transgenic (Tg) deletion mouse model, an Epstein Barr Virus LMP2A transgene integrated with $\sim 80$ copies into mouse chromosome 7B/C and created an $\sim 5 \mathrm{Mb}$ deletion of the mouse region homologous to the human PWS/AS genes (see Fig. 1A,B) [7]. As in human, the phenotype of the deletion mouse model depends on the parental origin: paternal or maternal inheritance of the Tg-deletion, respectively, results in the TgPWS mouse model characterized by severe neonatal hypoglycemia and early lethality [9] or in TgAS mice with a mild neurobehavioral phenotype and late onset obesity [7].

Previous imprinted gene expression studies and fluorescence in situ hybridization (FISH) showed that the Tg insertion-deletion comprised all of the orthologs of PWS and AS imprinted genes and of several flanking nonimprinted genes [Fig. $1 \mathrm{~A} ;[7,13,14]]$. In addition, brain microarray and quantitative gene expression analyses confirmed the loss of expression of several imprinted genes (Snurf-Snrpn, Ndn, Magel2, Mkrn3) and 50\% reduced expression of two non-imprinted loci (Herc2, Cyfip1) in
TgPWS mouse brain, and demonstrated that an unidentified non-imprinted locus within the deletion acted in trans to regulate a chromosome $18 \mathrm{~B} 3$ gene expression domain [15]. To define the exact characteristics of the transgene insertion-deletion TgPWS/TgAS mouse model, we have now determined the extent of the deletion and the effect of the Tg-insertion and deletion on expression of flanking genes. We delineated the deletion breakpoint positions between Siglec-H and Luzp2 at the centromeric end and within intron 4 of Chrna7 at the telomeric end. Most importantly, we describe tissue-specific positional effects of the Tg insertion and/or deletion on Luzp2 and Chrna7 expression which are likely due to the presence or absence of specific enhancer elements.

\section{Results \\ FISH refines the TgPWS/TgAS deletion extent and identifies a centromeric breakpoint}

We used FISH with BAC probes flanking the mouse PWS/ AS-homologous domain in chromosome 7B/C (Fig. $1 \mathrm{C}, \mathrm{D})$ to define the centromeric and telomeric extent of the TgPWS/TgAS deletion. To identify both chromosome 7 homologues in our FISH experiments using splenocytes from TgAS mice or fibroblasts from TgPWS mice, we cohybridized BAC RP22-434N7 from the Tyr gene locus at position $74.6 \mathrm{Mb}$ (chromosome 7E1), which is intact in TgPWS and TgAS mice (Fig. 2, and data not shown) [7].

At the centromeric end of the deletion, BAC RP24-354P8 spanning the Siglec-H-D7Mit70-Tubgcp5 region $(43.3 \mathrm{Mb}$, Fig. 1C) is deleted from one chromosome 7 in TgAS mice (Fig. 2A). Additionally, BACs RP23-256L9 ( $p$ gene exons 10-24; $44 \mathrm{Mb}$ ) and RP23-195C6 (Herc2-Nipa1; 43.6 Mb) are within the TgPWS/TgAS deletion (Fig. 1A, Fig. 2B, and data not shown) as expected based on their map position. In contrast, BAC RP24-426A19 from 50 kb 3' of Luzp2 (42.9 Mb; Fig. 1C) shows a weak positive signal on one chromosome 7 homologue in TgAS mice (Fig. 2C), suggesting a partial deletion and detection of the centromeric breakpoint in TgAS mice.

At the telomeric end of the deletion, BAC RP23-266F22 (49.4 Mb; Fig. 1D) spanning four mouse PWS-region imprinted genes, including Frat3, Mkrn3, Magel2 and $N d n$, was previously found to be deleted in TgPWS mice [13]. Extending telomeric from Frat3 towards Chrna7 (Fig. 1D), BACs RP23-100P8, RP23-199N11 and RP23-140J4 were all deleted in TgPWS mice (Fig. 2D, and data not shown). In contrast, BACs RP23-5O6 from the Klf13 region (50.9 Mb; Fig. 1D, Fig. 2E) and RP24-215K5 from 5' Cezanne2 (Fig. 1D; data not shown) were intact in TgPWS/TgAS mice. Two other BACs mapping more telomeric, RP2376F18 (Rlbp1 locus, 66.4 Mb) and RP23-441D12 (Il-16 locus, $70.8 \mathrm{Mb}$ ), were also intact in TgPWS mice (data not shown). Intriguingly, BAC RP23-16A4 spanning Chrna7 


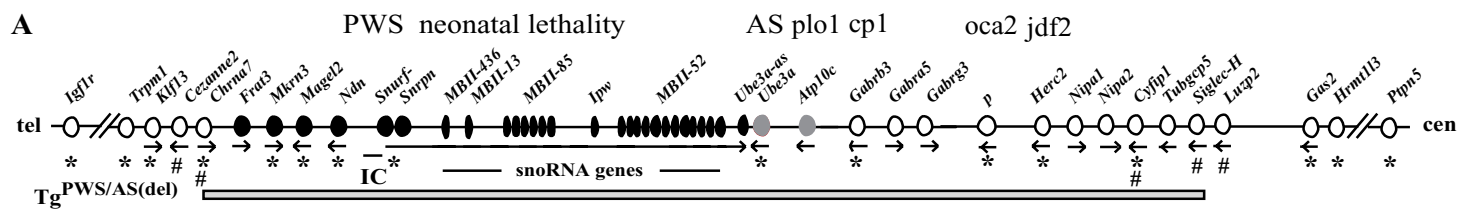

B

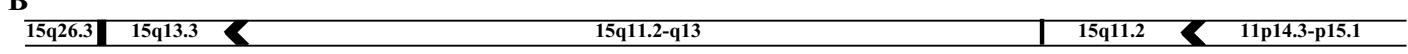

C
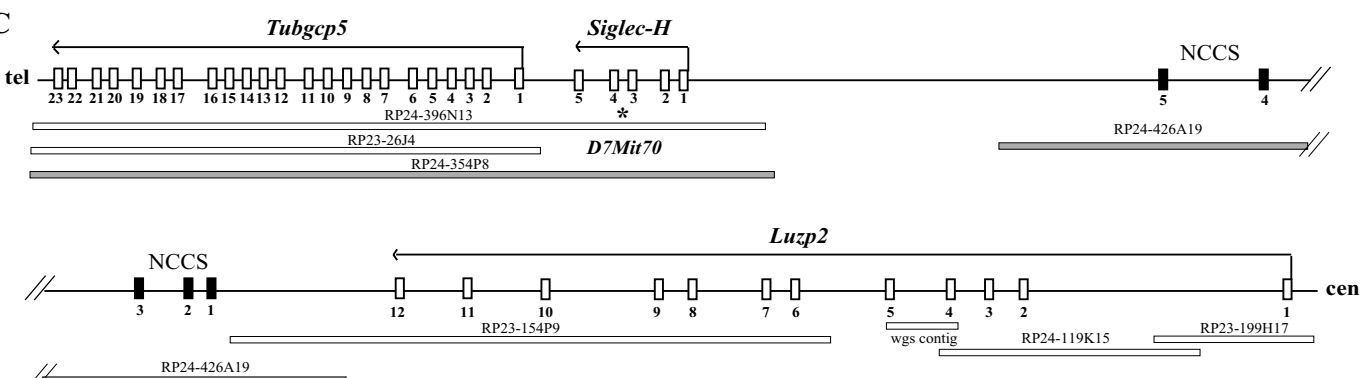

D
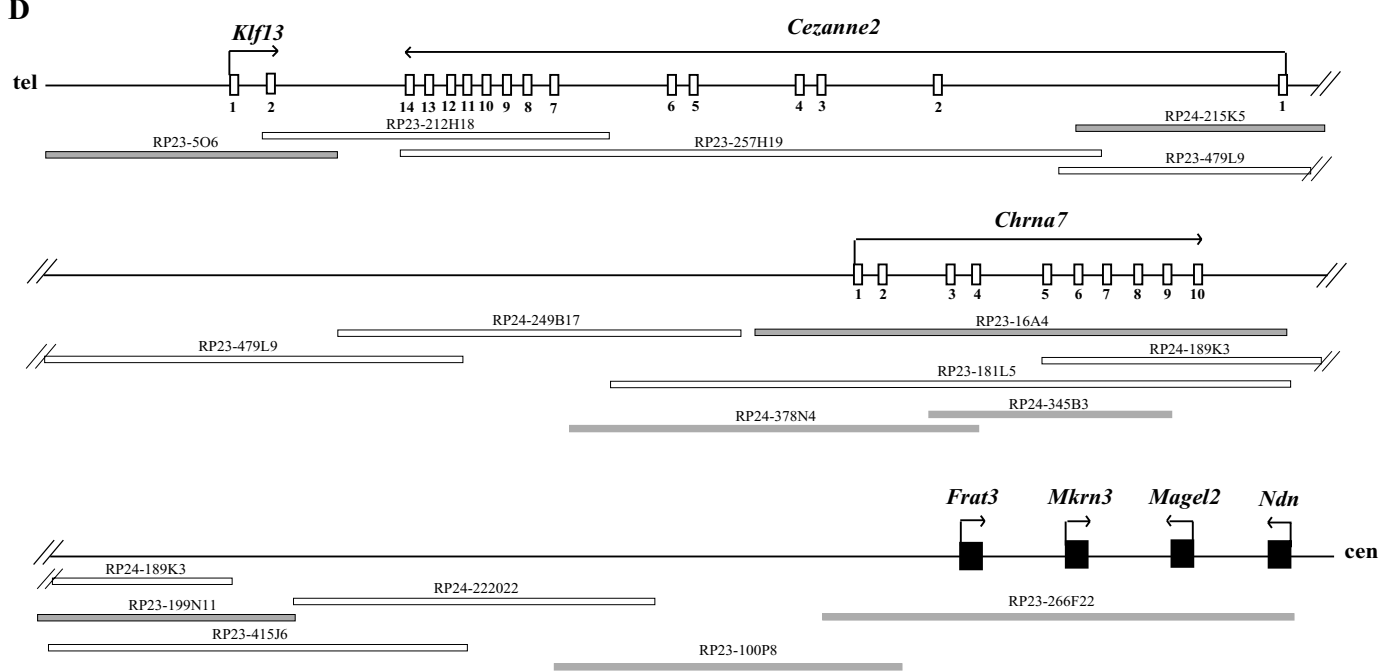

Figure I

Genetic and physical maps of mouse chromosome 7B/C. (A) The mouse PWS/AS-homologous region and flanking genes. Symbols are: circles, protein-coding genes; ovals, RNA-coding genes; black, paternally-expressed; grey, maternally-expressed; white, biparentally-expressed; line arrows, transcriptional orientation of genes; IC; imprinting center; ploI, $p$ locus-associated obesity; cp I, cleft palate I; oca2, oculocutaneous albinism type II ( $p$, pink-eyed dilution); jdf2, juvenile development and fertility 2; cen, centromere; tel, telomere; *, genes represented on the Gene chip MG_U74Av2 (see Table I); \#, genes analyzed by QRT-PCR; horizontal grey bar, extent of the TgPWS/TgAS 5 Mb deletion. The first 3 genes and one more distant gene extending out from each of the centromeric and telomeric deletion breakpoints are also shown. (B) Human synteny for genes from the mouse chromosome 7B/C domain. The human chromosome locations for each orthologous mouse gene shown in (A) are given. Arrowheads represent rodent-specific gene duplications for which no ortholog is found in human. (C) BAC contig across the TgPWS/TgAS centromeric deletion breakpoint region. Symbols are: white boxes, exons; line arrows, transcriptional orientation of genes; white bars, BACs, and one "wgs" (whole genome shotgun) contig; grey bars, BACs used as FISH probes; black boxes, Non-Coding Conserved Sequences (NCCSs) in RP24-246AI9; *, D7Mit70. The interval from Luzp2 through Siglec-H and Tubgcp5 is shown. (D) BAC contig across the TgPWS/TgAS telomeric deletion breakpoint region. Symbols are as for Fig IC, except: black boxes, four intronless imprinted genes. The interval from the telomeric end of the $2 \mathrm{Mb}$ imprinted gene domain through Chrna7 to Cezanne2 and KIfl 3 is shown. 

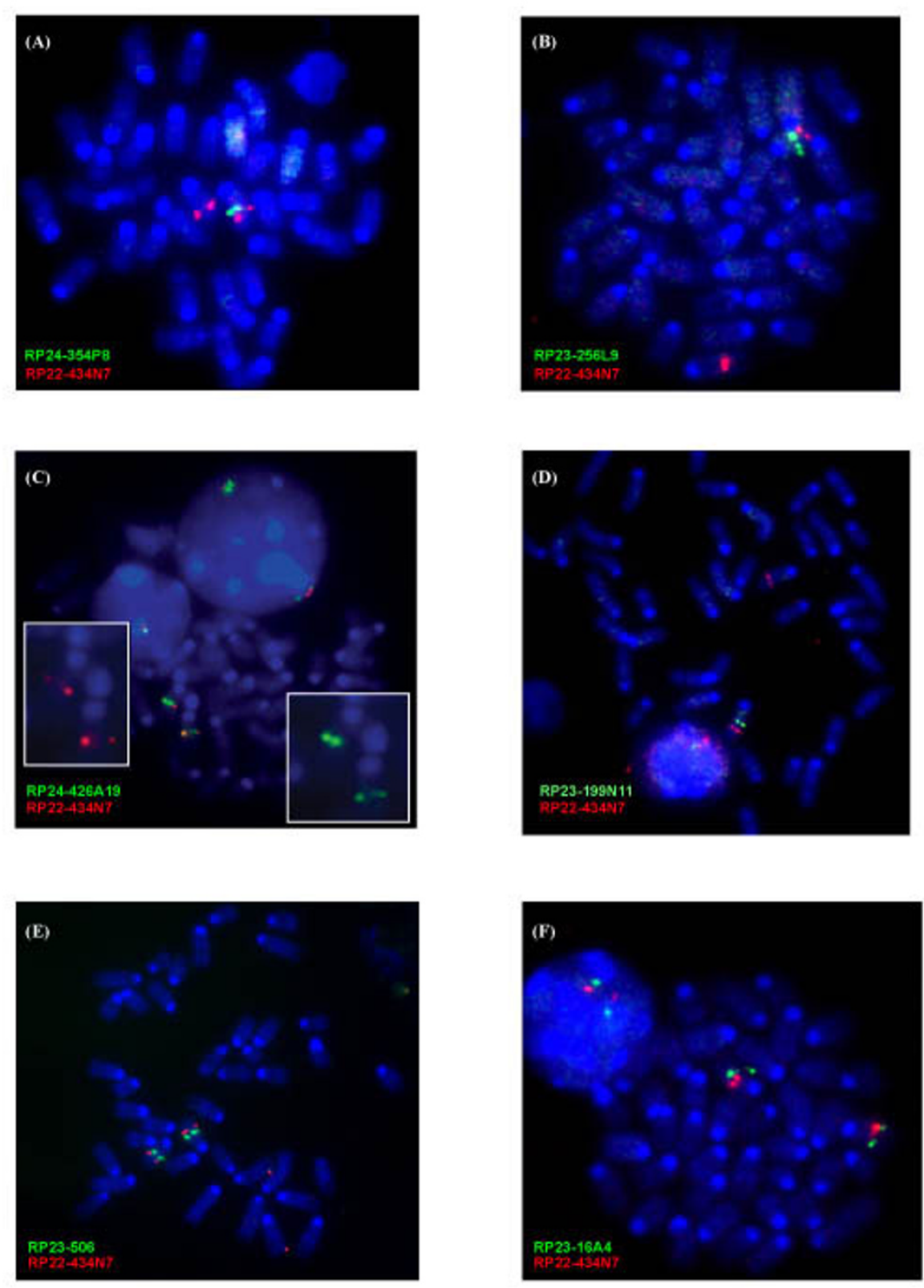

\section{Figure 2}

Fluorescence in situ hybridization (FISH) maps the TgAS centromeric and telomeric deletion extent. In each case, BAC RP22-434N7 from the chromosome 7 Tyrosinase (Tyr) locus (74.6 Mb; $44.0 \mathrm{cM}$ ) was hybridized as a control and is shown as a red signal. All chromosome 7B/C BACs used as probes are shown as green signals while chromosomes are stained with DAPI (blue). (A) BAC RP24-354P8 spanning the region including Siglec-H and Tubgcp5 shows a single-chromosome 7 signal indicating the deletion of this locus in TgAS splenocytes. (B) RP23-256L9 spans the $p$ locus (exons 10-24) and is also deleted in TgAS mice. (C) RP24-426Al9 spans the region 3' of Luzp2 and detects a weak signal on one chromosome 7 homologue, suggesting partial deletion and detection of the centromeric breakpoint in the mutant mice. The two insets show the images for individual probes. (D) RP23-I99N I I covers part of the region between Frat3 and Chrna7 and is deleted in TgAS mice. (E) RP23-506 for the KIfI 3 locus is intact in the deletion mice. (F) RP23- I 6A4 spans all 10 exons of Chrna7 and shows an apparently intact signal in TgAS mice. 
(50.1 Mb; Fig. 1D) appears based on hybridization signal intensity to be fully intact in TgPWS mice (Fig. 2F). Nevertheless, molecular data described below indicate that about half of 16A4 is deleted in TgPWS/TgAS mice; it is likely that these ostensibly contradictory data are explained by the relative concentration of unique and repetitive DNA sequences at the telomeric and centromeric ends of BAC 16A4 (5' and 3' ends of Chrna7, respectively) as well as the difficulty in quantifying FISH signals.

\section{Microarray and QRT-PCR analyses further define the TgPWS/TgAS deletion extent}

Previous analysis of brain global gene expression in 5 TgPWS and 5 wildtype (WT) mice at P1 using a MGU74Av2 gene chip array (Affymetrix) which assayed 12,000 genes and ESTs, demonstrated that all 4 paternally-expressed mouse PWS-region imprinted genes present on the array (Snurf-Snrpn, Ndn, Magel2 and Mkrn3) have dramatically reduced expression [15]. Additionally, mRNA levels for non-imprinted genes Herc2 and
Cyfip1 were 50\% in TgPWS compared with WT [15]. To assess the extent of the TgPWS/TgAS deletion and the potential effect of the transgene-insertion deletion on expression of flanking genes, we used data mining from several genome databases and analysis of several megabases of genome sequence to identify all genes in these regions, determined which of these were on the MGU74Av2 gene chip, and then reanalyzed the brain microarray data from reference [15]. Although a 3-4 Mb "gene desert" lies just proximal of Tubgcp5, we identified 9 genes on the chip in the $9.1 \mathrm{Mb}$ region centromeric of Tubgcp5, a locus which FISH data above had shown was deleted, of which four genes were not detectable in either TgPWS or WT brain at P1 (Table 1). Five genes (Gas2, Hrmt1l3, E2F8, Ptpn5, and Tsg101) were detectable and showed no change in TgPWS compared to WT mouse brain (Table 1), indicating that the TgPWS/TgAS deletion does not affect any of these genes (Fig. 1A). Telomeric of Frat3, an imprinted gene deleted in TgPWS mice [13], nine (Klf13, BB128963, Mcee, Apba2, Tjp1, Snrp2a, H47, Mef2a, and

Table I: Brain microarray data for mouse chromosome 7B2-C genes ${ }^{\mathrm{a}}$ in TgPWS vs. WT mice.

\begin{tabular}{|c|c|c|c|c|c|}
\hline \multirow[t]{2}{*}{ GenBank } & \multirow[t]{2}{*}{ Affy ID } & \multirow[t]{2}{*}{ Gene } & \multicolumn{2}{|c|}{ mRNA levelb } & \multirow[t]{2}{*}{ Chr. 7 location (Mb and/or $\mathrm{cM}$ ) } \\
\hline & & & TgPWS & WT & \\
\hline \multicolumn{6}{|c|}{ I. Genes centromeric of the TgPWS/TgAS deletion } \\
\hline \multirow[t]{2}{*}{ NM 008087} & 94337_at & Gas2 & $4.3(3 / 5)$ & $5.0(2 / 5)$ & $39.3 \mathrm{Mb} ; 26.8 \mathrm{cM}$ \\
\hline & 94338_g_at & Gas2 & $43.1(5 / 5)$ & $42.92(5 / 5)$ & as above \\
\hline AK049836 & 97539_at & Hrmt II3 & $50.2(5 / 5)$ & $44.5(4 / 5)$ & $37.25 \mathrm{Mb}$ \\
\hline NM 016865 & |0367|_at & Htatip2 & $23.8(0 / 5)$ & $21.0(0 / 5)$ & $37.2 \mathrm{Mb}$ \\
\hline \multirow[t]{2}{*}{ AY957576 } & 103202_f_at & E2F8 & $35.3(4 / 5)$ & $30.4(4 / 5)$ & $36.3 \mathrm{Mb}$ \\
\hline & 103204_r_at & E2F8 & $27.4(1 / 5)$ & $21.6(2 / 5)$ & as above \\
\hline$\underline{N M \quad 013808}$ & 103084_at & Csrp3 & $4.6(0 / 5)$ & $3.2(0 / 5)$ & $36.3 \mathrm{Mb}$ \\
\hline NM 013643 & 100406_at & Ptpn5 & $399.8(5 / 5)$ & $406.1(5 / 5)$ & $34.5 \mathrm{Mb}$ \\
\hline NM 016855 & 95304_at & Attp & $8.5(0 / 5)$ & $8.7(0 / 5)$ & $34.3 \mathrm{Mb}$ \\
\hline NM 021884 & 94809_at & TsglOI & $56.5(5 / 5)$ & $56.6(5 / 5)$ & $34.3 \mathrm{Mb}$ \\
\hline NM 013580 & 93103_at & Ldh3 & $1.7(0 / 5)$ & $1.3(0 / 5)$ & $34.3 \mathrm{Mb} ; 23.5 \mathrm{cM}$ \\
\hline \multicolumn{6}{|c|}{ II. Genes telomeric of the TgPWS/TgAS deletion } \\
\hline NM 007390 & 101131_at & Chrna7 & $86.8(0 / 5)$ & $87.1(0 / 5)$ & 50.I Mb; $30.0 \mathrm{cM}$ \\
\hline NM 021366 & 160617_at & KIfI 3 & $145.2(5 / 5)$ & $155.6(5 / 5)$ & $50.9 \mathrm{Mb}$ \\
\hline$\overline{N M \quad 018752}$ & $10225 \mid$ at & Trpml & $77.7(1 / 5)$ & $56.7(0 / 5)$ & $51.2 \mathrm{Mb} ; 27.0 \mathrm{cM}$ \\
\hline BC055074 & 93235_at & $B B / 28963$ & $51.3(5 / 5)$ & $46.0(5 / 5)$ & $51.3 \mathrm{Mb}$ \\
\hline NM 026483 & 104022_at & MphosphI0 & $35.9(0 / 5)$ & $34.0(0 / 5)$ & $5 \mathrm{l} .4 \mathrm{Mb}$ \\
\hline NM 028626 & 102022_at & Mcee & $79.6(5 / 5)$ & $81.1(5 / 5)$ & $5 \mathrm{I} .4 \mathrm{Mb}$ \\
\hline NM 007461 & 92727_at & Apba2 & $433.9(5 / 5)$ & $455.8(5 / 5)$ & $5 \mathrm{I} .5 \mathrm{Mb} ; 25.5 \mathrm{cM}$ \\
\hline NM 009386 & 99935_at & Tjp I & $185.3(5 / 5)$ & $142.5(5 / 5)$ & $52.3 \mathrm{Mb} ; 28.5 \mathrm{cM}$ \\
\hline D50060 & 101196_at & Pace4 & $78.3(0 / 5)$ & $71.2(0 / 5)$ & $52.9 \mathrm{Mb} ; 28.5 \mathrm{cM}$ \\
\hline NM 021336 & $101506 \_a t$ & Snrpal & $|3| .2(5 / 5)$ & $126(5 / 5)$ & $53.1 \mathrm{Mb}$ \\
\hline NM 024439 & 94245_at & H47 & $|4| .8(5 / 5)$ & $144.2(5 / 5)$ & 53.I Mb; $28.5 \mathrm{cM}$ \\
\hline NM 053080 & 98372_at & Aldh la3 & $13.3(0 / 5)$ & $9.8(0 / 5)$ & $53.4 \mathrm{Mb}$ \\
\hline NM 194070 & 93852_at & Mef2a & $399.9(5 / 5)$ & $417.4(5 / 5)$ & $54.3 \mathrm{Mb} ; 33.0 \mathrm{cM}$ \\
\hline AF056187 & 102224_at & lgfIr & $381.6(5 / 5)$ & $339.6(5 / 5)$ & $55.0 \mathrm{Mb} ; 33.0 \mathrm{cM}$ \\
\hline
\end{tabular}

a The primary microarray data from this experiment were reported in reference 15 . As summarized in Fig. IA, reference 15 included the data for the imprinted genes within the TgPWS/TgAS deletion that are on the MG-U74Av2 chip (Snrpn, Ndn, Magel2, Mkrn3 and Ube3a) and for nonimprinted genes within the deletion (Cyfipl, Herc2, and Gabrb3, although the latter transcript was not detectable; the $p$ gene is also represented on the chip although not detectable in TgPWS or WT mice, and not previously reported). Additionally, a cluster of chromosome I8B3 genes were found to be abnormally expressed in TgPWS mice [15]. ${ }^{\circ}$ mRNA levels are presented as the medians of Signal (relative abundance of a transcript), for TgPWS $(n=5)$ and WT $(n=5)$, with the number of calls as "detected" out of 5 comparison experiments in parentheses. 


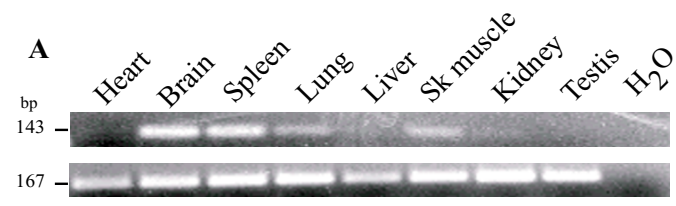

B

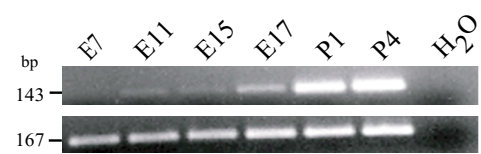

C

D
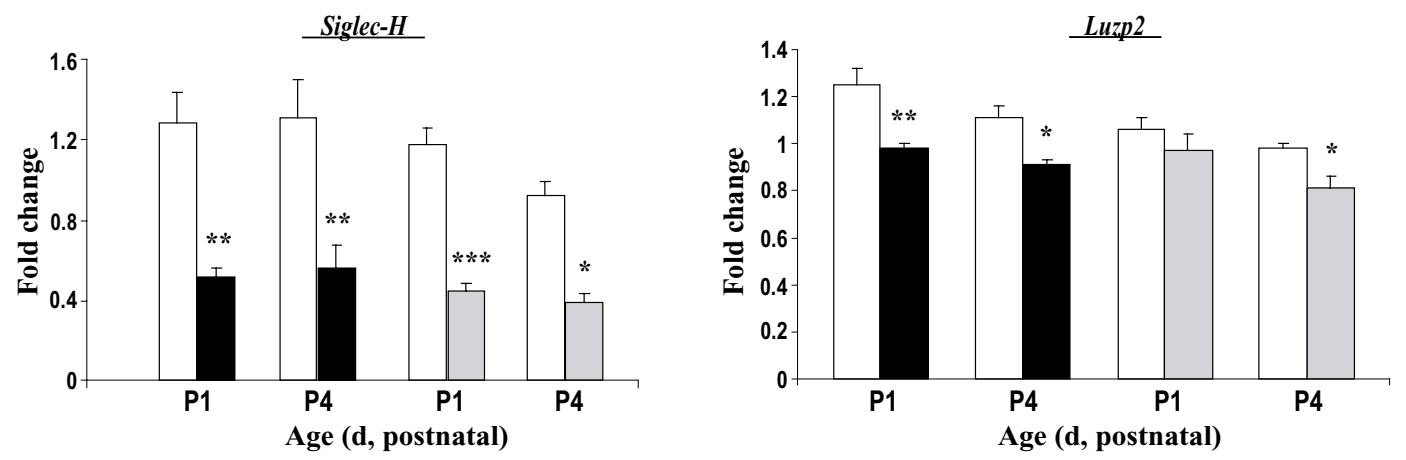

$\mathbf{E}$

$\mathbf{F}$
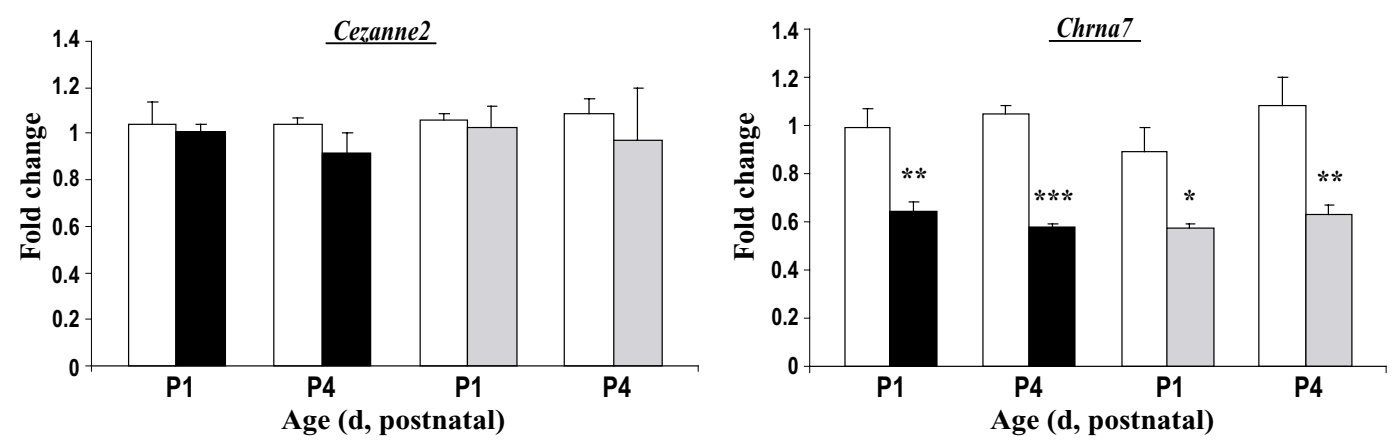

Figure 3

Gene expression analyses for loci at or near the TgPWS/TgAS deletion breakpoints. (A) Tissue expression of Siglec-H. RT-PCR using a cDNA mouse panel shows that Siglec-H (I43-bp band) is strongly expressed in adult brain and spleen, with moderate expression in lung and skeletal muscle tissues, but is low or absent in heart, liver, kidney and testis. RNApolll ( I67-bp) gene expression was used as a control for both (A) and (B). (B) Developmental Siglec-H expression. Very low or absent Siglec-H expression is detected by RT-PCR at E7-EI 5 with weak expression at late gestational stages (EI 7). After birth, SiglecH expression is robustly detected in brain at PI and P4. (C-F) Relative quantification of brain mRNA levels for (C) Siglec-H, (D) Luzp2, (E) Cezanne2 and (F) Chrna7 was performed by QRT-PCR in two groups of 5 TgPWS (black bars) or 5 TgAS (gray bars) and 5 WT (white bars) mice at PI and in 4 TgPWS or 4 TgAS and 4 WT mice at P4. All values are presented as the means \pm SE: $* P \leq 0.05$; ** $P \leq 0.00 I$; *** $P \leq 0.000$ I, significant differences between WT and TgPWS or TgAS at the indicated time points (Independent samples t-test). (C) Siglec-H mRNA levels are decreased by $\sim 2-$ fold in TgPWS and TgAS compared with WT at PI and P4. SiglecH mRNA levels in TgPWS vs. WT were $0.52 \pm 0.04$ vs. I.29 \pm $0.15(P=0.002)$ and $0.56 \pm 0.12$ vs. $I .31 \pm 0.19(P=0.01)$ at $P I$ and $P 4$, respectively. Siglec-H mRNA levels were $0.45 \pm 0.03$ in TgAS compared with $\mathrm{I} .18 \pm 0.08$ in WT at $P I(P=0.0002)$ and $0.39 \pm 0.04$ compared with $0.92 \pm 0.07$ at $P 4(P=0.0008)$. (D) Luzp2 mRNA levels in TgPWS vs. WT were $0.98 \pm 0.02$ vs. I $.25 \pm 0.07(P=0.009)$ and $0.9 \mathrm{I} \pm 0.02$ vs. I.II $\pm 0.05(P=0.0 \mathrm{I})$ at $\mathrm{PI}$ and $\mathrm{P} 4$, respectively. In TgAS vs. WT mice, mRNA levels were $0.97 \pm 0.07$ vs. $\mathrm{I} .06 \pm 0.05(P=0.3)$ at $\mathrm{PI}$ and $0.8 \mathrm{I} \pm 0.05$ vs. $0.98 \pm 0.02(P=0.01)$ at P4. (E) Cezanne2 shows no difference in expression in TgPWS or TgAS and WT mice. At PI, mRNA levels were I.0I \pm 0.03 for TgPWS vs. I.04 \pm 0.1 for WT $(P=0.8)$ and I.03 \pm 0.09 for TgAS vs. I.06 \pm 0.03 for WT $(P=0.7)$. At P4, Cezanne2 mRNA levels were $0.92 \pm 0.08$ for TgPWS vs. $1.04 \pm 0.03$ for WT $(P=0.2)$ and $0.97 \pm 0.23$ for TgAS vs. I.09 \pm 0.06 for WT $(P=0.6)$. (F) Chrna7 mRNA levels are $\sim 0.6$-fold in TgPWS and TgAS compared with WT mouse brain. At PI the mRNA levels were $0.64 \pm 0.04$ in TgPWS vs. $0.99 \pm 0.08$ in WT $(P=0.005)$ and $0.57 \pm 0.02$ in TgAS vs. $0.89 \pm 0.1$ in WT $(P$ $=0.01$ ), while at P4 mRNA levels for Chrna7 were $0.58 \pm 0.01$ for TgPWS vs. I.05 \pm 0.03 for WT $(P<0.0001)$ and $0.63 \pm 0.04$ for TgAS vs. $1.08 \pm 0.12$ for WT $(P=0.005)$. 

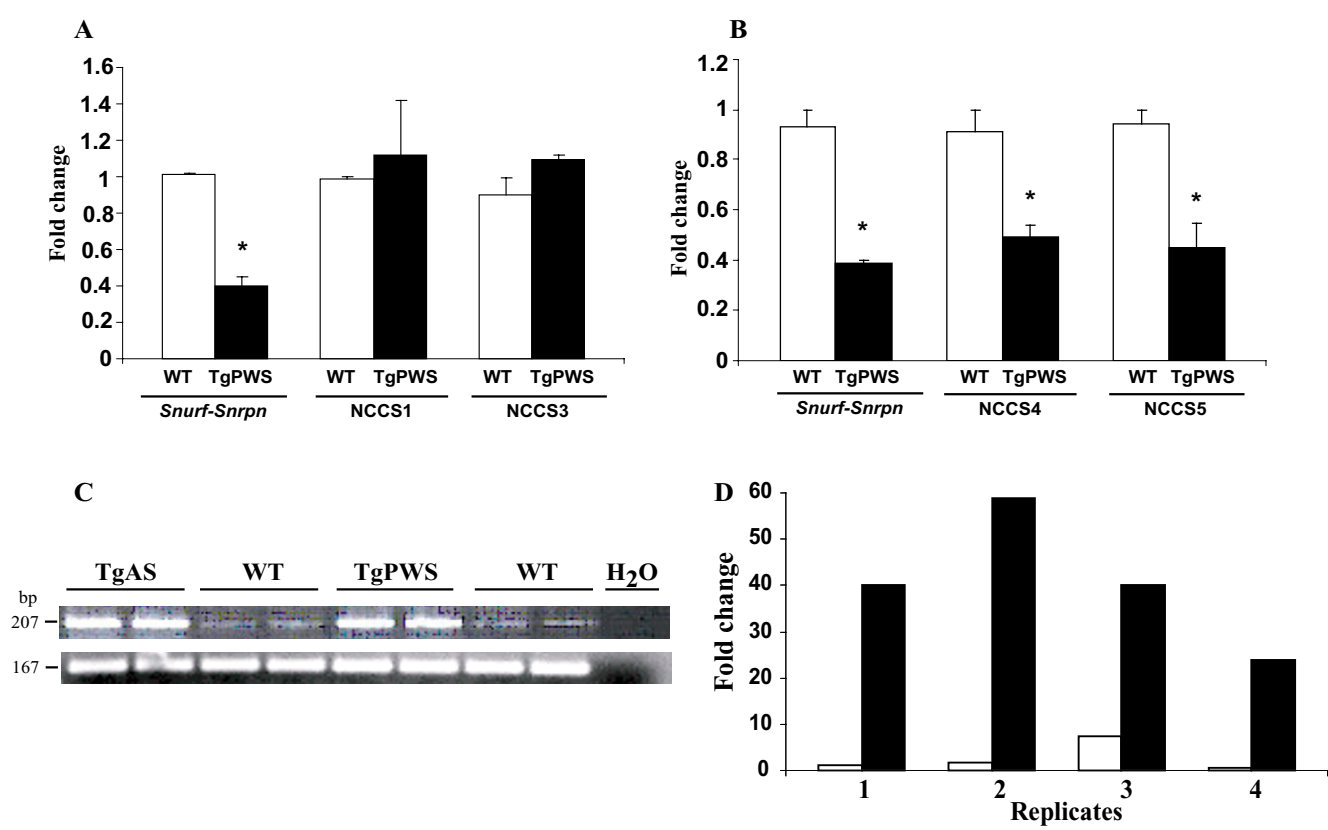

E

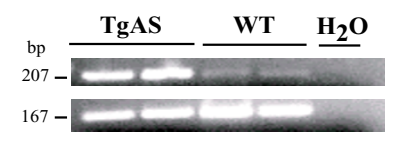

G

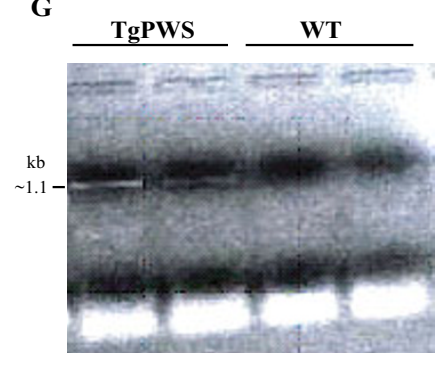

$\mathbf{F}$

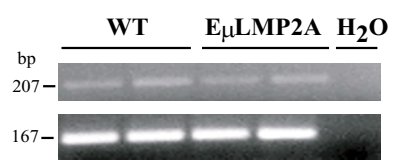

$\mathbf{H}$

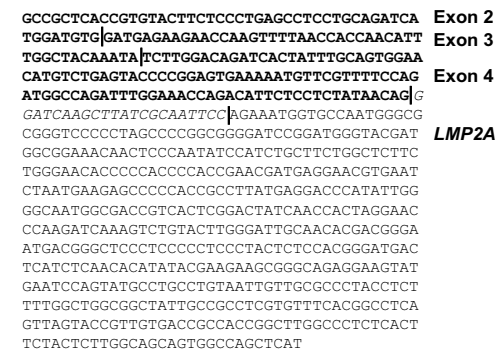

Figure 4

Mapping of centromeric and telomeric TgPWS/TgAS deletion breakpoints and tissue specific up-regulation of Chrna7. (A) Q-PCR for the Snurf-Snrpn promoter, NCCSI and NCCS3 relative to Gapdh intron I. The amount of Snurf-Snrpn DNA was I.0I \pm 0.01 for the WT compared with $0.40 \pm 0.05$ for the TgPWS samples, $P=0.006$. For NCCSI,

DNA fold was $0.98 \pm 0.02$ in WT vs. $1.12 \pm 0.30$ in TgPWS, $P=0.68$. For NCCS3, DNA fold was $0.90 \pm 0.09$ in WT compared with $1.09 \pm 0.03$ in TgPWS mice, $P=0.20$. (B) Q-PCR for NCCS4 and NCCS5 relative to Gapdh intron I. Snurf-Snrpn DNA-fold was $0.40 \pm 0.07$ in TgPWS vs. $0.90 \pm 0.1$ in WT mice, $P=0.02$. The amount of DNA for NCCS4 was $0.50 \pm 0.05$ in TgPWS and $0.90 \pm 0.1$ in WT mice, $P=0.05$. For NCCS5, DNA-fold was $0.45 \pm 0.1$ in TgPWS and $0.94 \pm 0.06$ in WT mice, $P$ $=0.05$. (C) Chrna7 expression (207-bp) is dramatically upregulated in liver of TgPWS and TgAS at PI compared with WT mice by RT-PCR. RNApolll expression (I67-bp) was used as a control in (C), (E) and (F). (D) Relative quantification by QRT-PCR of Chrna7 liver mRNA levels shows a $\geq$ I5-fold increased expression in TgPWS (black bars) vs. WT (open bars) mice. Individual values for Chrna7 mRNA expression of 4 TgPWS and 4 WT mice at PI are shown. The average mRNA expression was $40.76 \pm 14.30$ for TgPWS and $2.70 \pm 3.21$ for WT, $P=0.002$. (E) Up-regulation of Chrna7 expression in TgAS spleen compared with WT at PI by RT-PCR. (F) Chrna7 is normally expressed in PI liver from $E_{\mu} L M P 2 A$ transgenic mice compared with WT liver. (G) A Chrna7-LMP2A fusion transcript is identified by RT-PCR in TgPWS brain (lanes I,2) but not in WT (lanes 3,4). (H) DNA sequence of the Chrna7-LMP2A fusion cDNA. Vertical lines mark the limit between exons 2, 3 and 4 of Chrna7 (bold font) and the 5' end of LMP2A sequence, while the 22-nt in italics is a transgenespecific sequence at the $5^{\prime}$ end of the $L M P 2 A$ exon. 
Igf1r) of the fourteen genes extending out a further 5.6 Mb and on the microarray were detectable and all had comparable levels in TgPWS and WT mice (Table 1). Therefore, the Tg insertion-induced deletion does not affect gene expression over large regions outside the boundaries of the rearrangement.

We next assessed quantitative (Q) expression of genes that map in the proximity of the known deleted loci at the centromeric $[14,15]$ and telomeric $[14]$ ends of the deletion (Fig. 1A). At the centromeric end of the deletion, the Siglec- $H$ gene maps just proximal of Tubgcp 5 and is predicted to have five exons (Fig. 1C), with the rare feature of having the stop codon in the penultimate exon 4 [19]. Since there is no information regarding the pattern of expression for Siglec- $H[20,21]$, we first assessed this using RT-PCR (Fig. 3A,B). In agreement with the general signaling function of Siglec genes in the haemopoietic, immune and nervous systems [22], Siglec- $H$ was predominantly expressed in brain and spleen (Fig. 3A). Less robust expression was seen in lung and skeletal muscle, while there was little or no amplification in heart, liver, kidney and testis (Fig. 3A). A postnatal role for Siglec-H expression was suggested by the lack of amplification at early embryonic stages with increased expression after birth (Fig. 3B), as also found for ESTs in Unigene (data not shown).

By QRT-PCR, Siglec- $H$ showed 2-fold decreased mRNA levels in the brain of both TgPWS and TgAS mouse models at $\mathrm{P} 1$ and $\mathrm{P} 4(P<0.01$; Fig. $3 \mathrm{C})$, indicating that this gene appears to be within the TgPWS/TgAS deletion (Fig. 1A). It may also be noted that the 0.5 -fold expression level in TgPWS and TgAS mice compared with WT indicates that Siglec- $H$ is not an imprinted gene. The next gene centromeric of this, Luzp2, showed $\sim 0.8$-fold decreased mRNA levels in TgPWS brain at P1 and P4 and in TgAS brain at P4 $(P<0.05)$ (Fig. 3D). At P1 in TgAS, Luzp2 brain mRNA levels were decreased to 0.91 -fold $v$ s. WT $(P=0.3)$. Combined, the presence of the TgPWS/TgAS deletion resulted in a Luzp2 mRNA level in neonatal brain that is significantly $(0.83$-fold, $P=0.0002)$ less than in WT. We conclude that the Luzp2 gene is intact at the centromeric end of the TgPWS/TgAS deletion but suggest a model in which a neuronal regulatory element is deleted that accounts for $\sim 33 \%$ of Luzp2 expression from the deleted allele (see Discussion for a description of this and alternative models).

At the telomeric end of the deletion, expression of Cezanne2, which maps just centromeric of Klf13 (Fig. 1D), was unchanged by QRT-PCR in TgPWS and TgAS brain compared with WT mice (Fig. 3E), and thus this gene is intact in the TgPWS/TgAS deletion mouse model. The only remaining gene between Cezanne2 (intact in TgPWS/ TgAS mice) and Frat3 (deleted [13]) is Chrna7 (Fig. 1D), suggesting that the deletion breakpoint might lie in the vicinity of this gene. Chrna7 is orientated with a telomeric 5 ' end (Fig. 1D). Using QRT-PCR for Chrna7 and primers spanning exon 2 to exon 3, we found $\sim 1$.7-fold decreased Chrna7 mRNA levels in both TgPWS and TgAS brain at P1 and P4 $(P<0.05)$ (Fig. 3F). Since the 5 ' end of Chrna7 is not deleted in the TgPWS/TgAS mouse model (see below, which is also consistent with the normal FISH signal for BAC RP23-16A4 reported above), the finding of $\sim 60 \%$ total mRNA levels suggests that only $\sim 20 \%$ of the usual level of Chrna7 mRNA transcripts are generated from the deleted chromosome in TgPWS and TgAS mouse brain. The $80 \%$ reduction in expression in cis suggests that the deletion removes essential regulatory sequences (see Discussion).

\section{Fine-mapping of the centromeric TgPWS/TgAS deletion breakpoint}

The gene expression data described above suggest that Siglec-H is deleted in TgPWS and TgAS mice while Luzp2 is intact but shows slightly yet significantly reduced expression levels. In order to further delineate the centromeric breakpoint of the deletion and a potential mechanism for the $\sim 33 \%$ reduction in Luzp2 expression from the deletion allele, we examined the genome sequence of BAC RP24426A19 that lies between Siglec-H and Luzp2 (Fig. 1C) and that was identified above by FISH as partially deleted in TgPWS/TgAS mice. There are no exons for any gene encoded in this BAC. Nevertheless, using BLAST of RepeatMasked RP24-426A19 DNA sequence against the non-redundant GenBank database, extending from the centromeric end of BAC RP24-426A19 we identified five non-coding conserved sequences (NCCS1-5) at positions $\sim 30.1,39.9,68.8,107.3$, and $134.5 \mathrm{~kb}$, respectively (Fig. 1C). NCCS1-5 are 250-bp, 68-bp, 188-bp, 104-bp, and 157 -bp in length and show $82 \%, 91 \%, 81 \%, 82 \%$ and $83 \%$ identity with human, respectively. As in mouse, the five NCCS elements in human are located adjacent to LUZP2 and map in chromosome 11p14.3 (Fig. 1B). All five NCCS elements are likely to be involved in the regulation of $L U Z P 2$, while other flanking genes are unlikely to be regulated by or to relate to NCCS1-5, as the next most centromeric gene (Gas2) maps $3.2 \mathrm{Mb}$ from Luzp2 while the genes more telomeric are either non-syntenic in human (Fig. 1B), mapping to human chromosome 15q, or represent a rodent-specific acquisition (Siglec-H; see Discussion).

PCR primers were designed from four of the NCCS elements and Q-PCR of DNA from TgPWS and WT mice was used to examine the relative amount of each NCCS with respect to intron 1 of Gapdh, which is intact in all mice studied. As a deletion control, we similarly examined by Q-PCR the Snurf-Snrpn promoter, which as expected showed a 0.4 to 0.44 -fold relative level in TgPWS vs. WT 
A
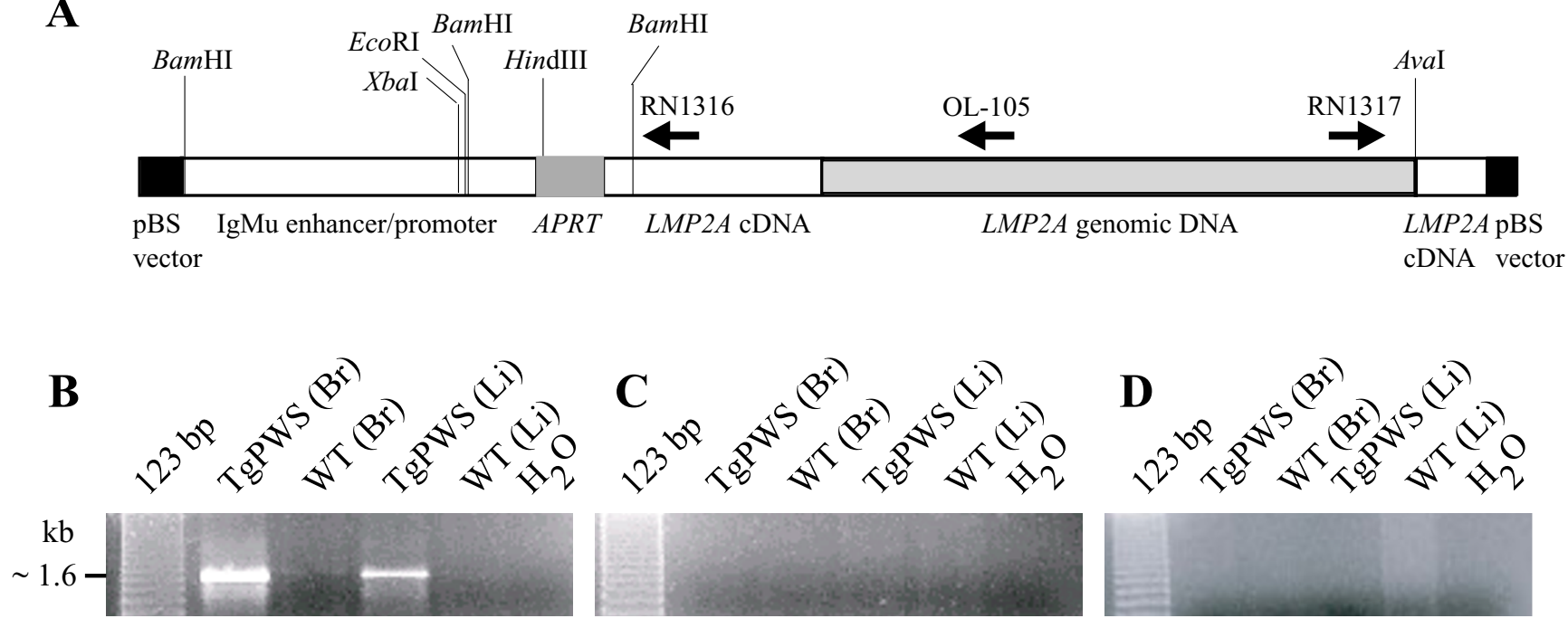

Figure 5

Orientation of the LMP2A transgene array. (A) LMP2A transgene structure. Restriction enzyme sites and primer location and orientation are shown. (B) LMP2A transgene amplification in TgPWS brain (Br) and liver ( $\mathrm{Li})(\mathrm{lanes} 2,4)$ using primers RNI3I6 and RNI3I7 compared with the WT controls (lanes 3,5). (C-D) The use of (C) RNI3I6 or (D) RNI3I7 alone fails to generate PCR products in TgPWS and WT brain and liver.

mice (Fig. 4A,4B). Whereas there was no difference between TgPWS and WT mice for NCCS1 and NCCS3 (Fig. 4A), indicating that NCCS1, NCCS2 and NCCS3 are intact in TgPWS mice, Q-PCR for NCCS4 and NCCS5 identified 1.8-fold and 2.1-fold lower levels for TgPWS $v$ s. WT, respectively (Fig. 4B), indicating that these are deleted from one allele in TgPWS mice. Therefore, the centromeric breakpoint of the TgPWS/TgAS $5 \mathrm{Mb}$ deletion lies in the $38.5 \mathrm{~kb}$ region between NCCS3 and NCCS4.

\section{Up-regulation of Chrna7 allows mapping the telomeric TgPWS/TgAS deletion breakpoint}

In contrast to the results shown above for Chrna7 expression in brain, regular RT-PCR for Chrna7 in newborn liver showed a dramatic increased expression in TgPWS and TgAS compared with WT (Fig. 4C). Indeed, quantification of liver Chrna7 expression by QRT-PCR revealed $~ 15$-fold increased mRNA levels in TgPWS mice $(P=0.002)$ (Fig. 4D). A similar up-regulation of Chrna7 expression was found by RT-PCR in spleen tissues of TgAS mice (Fig. 4E). However, $\mathrm{E}_{\mu} \mathrm{LMP} 2 \mathrm{~A}$ control mice with B-cell lineage expression of $L M P 2 A$ showed normal expression of Chrna7 in newborn liver (Fig. 4F). Combined, these data on two different $L M P 2 A$ transgene models indicates that LMP2A expression in B cells does not upregulate Chrna7 in trans, and suggests that the increased expression of Chrna7 observed in liver and spleen from TgPWS and TgAS mice results from a cis effect of the transgene inser- tion. Since B-cell transcription of the LMP2A transgene in these models is driven from an immunoglobulin heavy chain gene promoter and enhancer $[7,16]$, we hypothesized that the latter enhancer was also driving transcription of the heterologous, endogenous Chrna7 promoter.

To test this hypothesis, we used an exon 2 forward primer for Chrna7 and a reverse primer (OL-105) from inside the Tg (Fig. 5A) to perform RT-PCR on cDNA from TgPWS $v s$. WT brain, which detected a $1.1 \mathrm{~kb}$ product specifically in TgPWS samples (Fig. 4G). The $1.1 \mathrm{~kb}$ band was cloned and sequenced, identifying it as a fusion transcript encoding Chrna7 exons 2-4 fused to the sense strand of the LMP2A transgene (Fig. $4 \mathrm{H}$ ). The latter result indicates that at least the telomeric copy of the Tg array is orientated in a 5'-3' telomere to centromere fashion. Furthermore, we can conclude that the $5^{\prime}$ end of the Chrna7 locus is intact and correctly splices through exon 4 but then splices into the LMP2A transgene. These data strongly suggest that the telomeric breakpoint of the TgPWS/TgAS $5 \mathrm{Mb}$ deletion is in the $38.1 \mathrm{~kb}$ Chrna7 intron 4 .

\section{Orientation of the LMP2A transgene array}

To fully understand how the transgene array might affect flanking gene expression, it is necessary to define the integrity of the array and the orientation of each transgene copy and to flanking genes. To characterize the transgene array, we amplified genomic DNA from TgPWS mice and 
WT controls using primers from within the transgene (Fig 5A). Amplification with either single primer (RN1316 or RN1317) did not lead to any product (Fig. 5C,D), indicating that there are no head-to-head or tail-to-tail copies of adjacent transgene inserts. In contrast, PCR using primers from adjacent copies of the transgene results in amplification of the LMP2A array in TgPWS DNA (Fig. 5B). These data indicate that the $\sim 80$ copies of the transgene [7] are all in the same tail-to-head orientation. Combined with the data described above on the sense strand fusion of Chrna7 and LMP2A cDNA sequences, we conclude that the entire Tg array is orientated in a 5'-3' manner from the telomeric to centromeric end.

\section{Discussion}

\section{A mouse model of PWSIAS class I deletions}

The TgPWS and TgAS mouse models were created by insertion of an LMP2A transgene array of $\sim 80$ copies [7] that fortuitously generated a deletion equivalent to those that occur in PWS and AS in the human. Although the mouse deletion was previously estimated as being $\sim 5 \mathrm{Mb}$ in length, genome sequence analysis indicates that the deleted segment could be as large as $6.8 \mathrm{Mb}$ (Siglec- $H$ to Chrna7 distance). However, there are a few gaps in the sequence, particularly in several duplicated genomic regions throughout the imprinted domain, and so the exact size of this interval remains unknown. Nevertheless, our previous [7,13-15] and present studies allow the conclusion that 13 imprinted and 11 non-imprinted genes are included within the mouse PWS/AS-deletion region (see Fig. 1A). In addition to spanning all the PWS- and AShomologous paternally and maternally expressed genes, respectively, the TgPWS/TgAS deletion in mice also includes the homologs of typically deleted non-imprinted genes (ie., Gabrb3, Gabra5, Gabrg3, p/Oca2, and Herc2) as well as those of the PWS/AS genes (Nipa1, Nipa2, Cyfip1 and Tubgcp5) that define the deletion as equivalent to human class I deletions $[1,4]$. Although the classic PWS or AS clinical phenotype is similar in both class I and class II deletions, respectively, a more severe neurobehavioral phenotype has been described for class I compared with class II deletion patients as well as for deletion $v s$. UPD patients in each syndrome $[23,24]$. The TgPWS and TgAS deletion mouse models may therefore prove useful to compare the neurobehavioral phenotype to the UPD or ID PWS [5,6] or Ube3a gene mutation AS [11,12] mouse models.

Although the TgPWS/TgAS mouse models show reduced expression of several other genes, including Siglec- $H$, Luzp2 and Chrna7, as compared to the human PWS and AS class I deletions, none of these additional genes are likely to contribute to the TgPWS and TgAS phenotypes. For example, since Siglec- $H$ is a recent evolutionary addition in the rodent genome [20], its 50\% reduction in expression levels due to hemizygosity at the centromeric end of the deletion is unlikely to have a phenotypic effect. Likewise, the $\sim 17 \%$ reduction in overall expression of Luzp2 observed in brain of TgPWS/TgAS mice is unlikely to be functionally important, since complete loss of Luzp2 does not lead to any specific phenotype [25]. Although Chrna7 is another gene partially deleted in TgPWS/TgAS mice and the human ortholog maps in 15q13, the latter is outside the PWS/AS deletions and the TgPWS/TgAS phenotype is unlikely to be affected by Chrna7 hemizygosity, since Chrna7 heterozygous knockout mice show no abnormal phenotypes and even Chrna7-/- null mice have only mild skin and reproductive phenotypes [26-29].

\section{Implications for evolutionary acquisition of new genes and breakpoint mechanisms}

Our study also characterized expression of a recently identified gene, Siglec- $H$, which belongs to the Siglec gene family encoding immunoglobulin-like lectins that act as extracellular receptors for sialic acid residues of glycan chains $[20,21]$. The majority of Siglec genes map in a single CD33 (Siglec-3)-related cluster in human 19q13 and the syntenic mouse chromosome $7 \mathrm{~B} 2$ region [20,21]. In contrast, we show here that Siglec- $H$, which is rodent-specific and has no primate ortholog [21], lies adjacent to the mouse PWS/AS-homologous region at a position $12.3 \mathrm{Mb}$ from its ancestral location. It seems likely that Siglec-H has similar postnatal immunological functions as other Siglecs, given its robust expression in spleen. However, in contrast with the restricted expression of most other Siglec genes to the haematopoietic and immune systems, Siglec$H$ is also transcribed at high levels in postnatal brain. Although conjectural, it is tempting to speculate that neuronal expression of Siglec- $H$ may have arisen due to an evolutionary positioning adjacent to single (Luzp2) [25] or clustered (ie., Tubgcp5, Cyfip1, Nipa2, Nipa1) [14] genes expressed at high levels in the nervous system, such that Siglec- $H$ transcription may be under control of a neuronal enhancer for one or more of these genes.

Intriguingly, both the centromeric and telomeric TgPWS/ TgAS deletion breakpoints map at or close to the positions of chromosomal evolutionary breakpoints, and at each of the latter two positions there are also rodent-specific gene duplication-insertions. At the centromeric end, the TgPWS/TgAS deletion breakpoint lies immediately adjacent to Siglec- $H$, which as discussed above arose in rodents by a genomic duplication from a precursor gene located $\sim 12 \mathrm{Mb}$ away. The evolutionary insertion of Siglec-H between Tubgcp5 and Luzp2 occurs right at the boundary of synteny with human chromosome 15q11.2-q13 and 11p14.3-p15.1, respectively (Fig. 1B). Moreover, the TUBGCP5 ortholog also lies within a few kilobases of a primate evolutionary breakpoint that translocated the TUBGCP5 - CYFIP1-NIPA2-NIPA1-HERC2-duplicon clus- 
A Neuronal cells/disrupted 3' of Luzp2

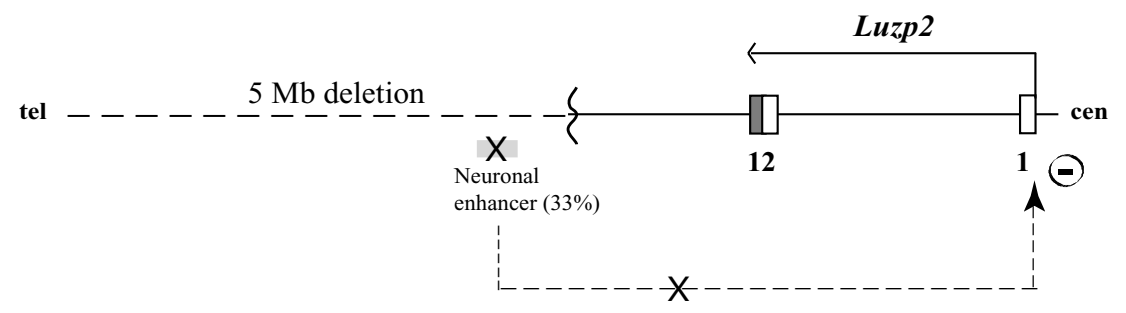

B B cells/disrupted Chrna 7

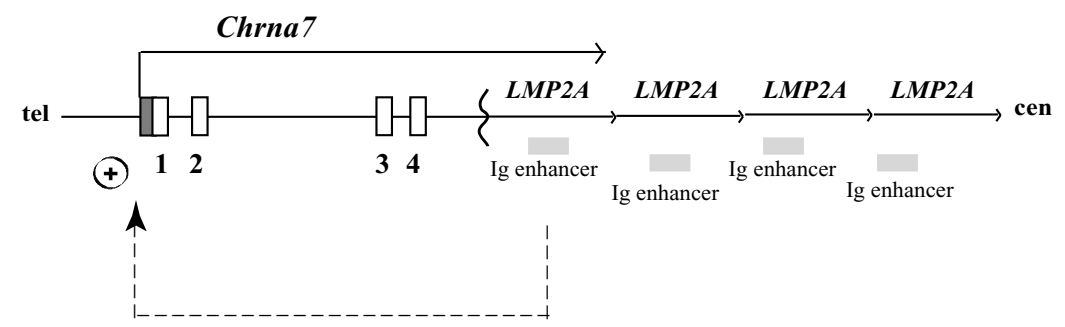

C Neuronal cells/intact Chrna7

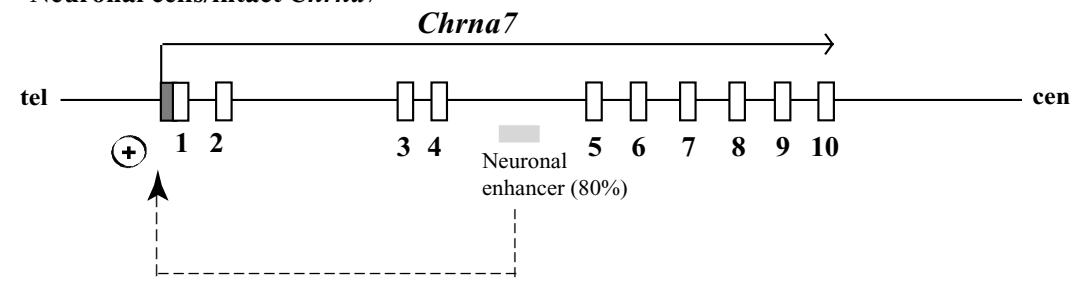

D Neuronal cells/disrupted Chrna 7

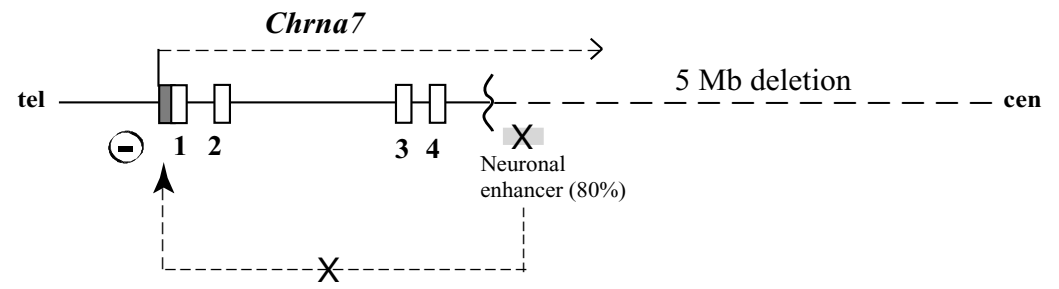

Figure 6

Enhancer models for tissue-specific expression of Luzp2 and Chrna7. (A) Deletion of a putative neuronal enhancer 3' of Luzp2 lowers expression levels of the gene. Symbols are: white boxes, exons; grey vertical box, 3'untranslated region (UTR); arrow, transcriptional orientation; zig-zag line, transgene insertion-deletion breakpoint; grey horizontal box, enhancer; dashed arrow, neuronal enhancer contributing $33 \%$ to the Luzp2 allelic promoter activity; X, block to enhancer function. Only the first and last exons of Luzp2 are shown. (B) Activation in TgPWS and TgAS mice of the Chrna7 promoter in cis by the immunoglobulin (Ig) enhancer from the LMP2A transgene active in B lymphocytes. Symbols are as for Fig. 6A, except: grey vertical box, 5'-untranslated region (UTR); dashed arrow, Ig enhancer upregulation of Chrna7 transcription. (C) Activation of the Chrna7 promoter in WT mice by a putative neuronal enhancer. The location of the enhancer is drawn arbitrarily but must map 3' to the TgPWS/TgAS deletion breakpoint in intron 4. Symbols are as for Fig. 6B, except: dashed arrow, neuronal enhancer for upregulation ( 80\%) of normal Chrna7 transcription. (D) Deletion of a neuronal enhancer in TgPWS and TgAS mice virtually silences Chrna7 expression in cis. Symbols are as for Fig. 6A and 6C. 
ter to $15 \mathrm{q} 11.2$ from an ancestral $15 \mathrm{q} 13$ location [14]. Although the telomeric TgPWS/TgAS deletion breakpoint within Chrna7 is not right at an evolutionary breakpoint, this gene also lies adjacent to an evolutionary breakpoint separating genes whose human orthologs map to the PWS imprinted domain in $15 \mathrm{q} 11.2$ and a point $8.7 \mathrm{Mb}$ away in 15q13.3 (Fig. 1B). At this position also, there are speciesspecific duplications in mouse and human, with the retrotransposed Frat3 limited to rodents [13] or the HERC2and flanking duplicons in primates $[5,14]$. Other authors have also noted the apparent congruity of chromosome rearrangement and evolutionary breakpoints [14,30-32], suggesting that currently unknown chromosomal structural features in these regions may play recombinogenic roles.

\section{Effect of the transgene or deletion on gene expression: mapping of putative neuronal enhancers}

Transgene insertions may be accompanied by other chromosomal rearrangements such as deletions (this paper; [33]), duplications [34], inversions [35], translocations [36], or combinations of rearrangements [36,37]. These events can induce alterations of gene expression over large chromosomal regions [33], or the transgene itself can do so [38]. Extensive analysis of genes flanking the TgPWS/ TgAS transgene insertion demonstrated no other rearrangements other than the PWS/AS-region deletion and that gene expression over large regions outside the immediate flanking genes was not affected. In contrast, expression from the first gene promoter outside either end of the transgene insertion-deletion was affected. For example, the 5' promoter and 3' end of Luzp2 are $600 \mathrm{~kb}$ and $110-$ $140 \mathrm{~kb}$ centromeric to the LMP2A transgene array-deletion breakpoint, respectively, and this gene shows a significantly reduced expression in TgPWS and TgAS mice. Although at present we cannot fully exclude three models that might lower the Luzp2 transcriptional level in cis in brain, which invoke either (i) a non-specific disruption of chromatin, (ii) a weak silencing effect from the transgene tandem array [45], or (iii) extended antisense transcripts from the transgene array given that the 5'-3' orientation of the LMP2A array is opposite to the Luzp2 direction of transcription, we feel that these are less likely than a fourth model that has additional experimental support. We propose that the 33\% reduction in expression of Luzp2 from the deletion allele is most likely explained by deletion of a neuronal enhancer (Fig. 6A). Consistent with this model, we identified two NCCS elements (NCCS4 and NCCS5) conserved in all eutherian mammals sequenced to date and deleted in TgPWS and TgAS mice, that represent strong candidates to be the neuronal enhancer. As noted above, these elements map to the homologous chromosome location in human but are almost $4 \mathrm{Mb}$ from the next most centromeric gene (Gas2) while other flanking genes in mouse are non-syntenic (Tubgcp5) or not present (Siglec-H) in human (Fig. 1B). Numerous NCCSs occur within mammalian genomes $[39,40]$, and while the majority of these have no known function, many do act as enhancer elements [41-43]. Future in vitro and in vivo studies [44] will be able to examine this enhancer model for Luzp2.

At the telomeric end of the deletion, expression of Chrna7 was dramatically upregulated in liver and spleen, but not brain, due to a tissue-specific positional effect of the transgene. We propose that the transgene immunogobulin $\mathrm{Mu}$ enhancer acts in B cells to promote transcription in cis from the heterologous Chrna7 promoter (Fig. 6B). Similarly, transgene regulatory elements $1 \mathrm{Mb}$ away have been shown to activate Sox9 [38]. Moreover, our data combined with cloning of a 5'-Chrna7-LMP2A fusion transcript clearly demonstrates that the Chrna7 promoter is intact and fully capable of high levels of enhancer driven activity on the deletion allele in TgPWS/TgAS mice.

Perhaps the most intriguing finding of this study was our serendipitous discovery that a putative neuronal enhancer for the Chrna7 gene maps within the telomeric end of the TgPWS and TgAS deletion. In TgPWS and TgAS mice, the Chrna7 mRNA level in brain from the deletion chromosome 7 falls to $20 \%$ of normal levels despite the fact that the $5^{\prime}$ promoter is not deleted. Non-enhancer models appear unlikely to explain this data: for example, models invoking (i) non-specific disruption of chromatin or (ii) a silencing effect from the transgene tandem array [45] are unlikely to account for the specific $\sim 80 \%$ reduction in $c i s$ expression levels in TgPWS and TgAS mouse brain, while allowing a dramatic activation of the same promoter in immune tissues, and, finally (iii) Chrna7 is transcribed from upstream and in the same orientation as the transgene array, so that an antisense mechanism is not valid. Therefore, we propose that sequences that map 3' of the deletion breakpoint in Chrna7 intron 4 are critical for Chrna7 expression in neurons (Fig. 6C), and it is the deletion of this putative neuronal enhancer that accounts for the reduced Chrna7 expression in brain of TgPWS and TgAS mice (Fig. 6D). Similar analyses of Chrna7 gene expression in other tissues of TgPWS and TgAS mice, such as the neuromuscular junction, heart, and skin, will allow further assessment of the tissue specificity of the proposed enhancer. Interestingly, our data in brain and other tissues also imply that the Chrna7 promoter in vivo only has basal transcriptional activity whereas for high levels of transcription, Chrna7 requires activation by a tissue-specific enhancer(s). In support of this model, we recently identified as candidate enhancer elements two NCCSs within Chrna7 intron 4, whereas no other conserved non-coding sequences other than the minimal promoter occur in a $412 \mathrm{~kb}$ domain starting $18 \mathrm{~kb} 5^{\prime}$ of Chrna 7 and extending 3 ' of the gene towards the mouse PWS-region imprinted 
domain (R.D.N., K.C., and M.S., unpublished data). We are currently mapping the Chrna7 NCCS elements against the TgPWS/TgAS deletion endpoints, while in vitro and in vivo approaches will be needed for analysis of function as candidate neuronal transcriptional enhancers and/or for other cell types.

\section{Conclusion}

In this study, we used a variety of molecular cytological and genetic technologies to map the transgene insertionchromosome deletion breakpoints of a mouse model of PWS and AS to $\sim 38 \mathrm{~kb}$ regions between the Luzp2 and Siglec- $H$ genes at the centromeric end and within Chrna7 intron 4 at the telomeric end, respectively. Gene expression analyses then allowed us to demonstrate that genes extending out a further 9.1- or 5.6-Mb centromeric or telomeric of the deletion, respectively, are not affected by either the deletion or insertion of the transgene tandem array. In contrast, transcription of genes at (Chrna7) or flanking (Luzp2) the transgene insertion-deletion breakpoints are affected by the disruption of normal chromosome architecture, with positional effects leading to up- or down-regulation dependent on the tissue-specificity and locations of enhancers within the transgene or putatively removed by the TgPWS/TgAS chromosome deletion. Using Luzp2 as an example, we demonstrate that analysis of phylogenetically conserved sequences and consideration of synteny allows the fine mapping of a putative neuronal enhancer element(s), while a similar model likely also applies for Chrna7. In human, CHRNA7 is of significant interest as a schizophrenia candidate gene with both genetic and functional support [46-48]. Nevertheless, the molecular basis of its candidacy remains uncertain since rare coding and in vitro characterized promoter variants in CHRNA7 $[49,50]$ may be insufficient to account for deficient function in vivo [51], particularly in light of our observations that expression of Chrna7 in vivo requires a major participation of enhancer function. Identification of a neuronal enhancer and further studies in the human to examine for genetic variation and potential mutations might provide an explanation for a role of CHRNA7 as a schizophrenia-susceptibility gene.

\section{Methods \\ Animals}

We used three mouse models all of which have an $L M P 2 A$ transgene insertion. In the TgPWS and TgAS models, the insertion of an $L M P 2 A$ transgene array replaced the PWS/ AS-homologous region in chromosome $7 \mathrm{~B} / \mathrm{C}[7,9,15]$ while the $\mathrm{E}_{\mu} \mathrm{LMP} 2 \mathrm{~A}$ mice have $\mathrm{B}$-cell lineage expression of LMP2A [16]. WT littermates were used as controls in each experiment. The University of Pennsylvania Institutional Animal Care and Use Committee approved all animal experiments. All animals were bred and genotyped as described $[7,16]$.

\section{BAC contig assembly and fluorescence in situ hybridization (FISH) studies}

Splenocytes from TgAS mice were cultured for $48 \mathrm{hr}$ in the presence of concanavalin A for stimulation of cell proliferation. After harvesting, cells were treated with hypotonic buffer $(0.075 \mathrm{M} \mathrm{KCl})$ for $15 \mathrm{~min}$, chromosomes were fixed with methanol-acetic acid (3:1) and spread onto slides [17]. For some probes, fibroblast cell lines from TgPWS mice were used. BAC DNA was labeled with biotin or digoxigenin and hybridized as previously described [18]. Image acquisition was performed using fluorescence microscopy, acquiring grayscale images using separate filters for each fluorophore followed by digital merging and pseudocoloring. BAC RP22-434N7, spanning the mouse chromosome 7 tyrosinase gene, was used as a control probe to identify both chromosome 7 homologues.

The chromosome 7B/C BAC contigs were generated by standard bioinformatics analyses using Ensembl [52] and BLAST [53]. The position and overlap of each BAC clone was determined by BLAST using gene cDNA/EST sequences, BAC end sequences, and STS sequences. Previously described BACs are RP23-266F22 [13], RP23256L9, RP23-195C6, RP24-396N13 and RP24-354P8 [14]. It may be noted that we previously incorrectly assumed the position of Trpm1 (formerly Mlsn1) [7], whereas analysis of genomic sequence data now clearly place this locus telomeric to the TgPWS/TgAS deletion (see Fig. 1A).

\section{Gene expression analyses \\ Microarray analysis}

Brain global gene expression was assessed in five TgPWS and five WT male CD-1 sibs at P1 using the Affymetrix Murine Genome (MG) U74Av2 Array. The microarray experiments and data processing, with identification of genes significantly altered in TgPWS brain, were performed previously (see also Table 1) [15]. In the present study, we reanalyzed the microarray data for genes identified from bioinformatics analyses (see above) as mapping adjacent to the TgPWS/TgAS deletion.

\section{QRT-PCR}

Brain tissues from four or five each of TgPWS, TgAS and littermate WT mice at P1 and P4 were used for most QRTPCR experiments. Liver Chrna7 expression was determined in four TgPWS and four WT mice at P1. QRT-PCR analysis was performed as described [15]. Briefly, total RNA was extracted using TRIzol (Invitrogen) and reversetranscribed using Superscript First-Strand Synthesis System (Invitrogen). For all reactions, each sample was loaded in triplicate and SYBR green was used as the fluorescent dye. Gapdh was used as an internal control. Relative quantification of gene expression was carried out using a PRISM 7000 Sequence Detection System (Applied 
Biosystems Inc.) and data was processed using a comparative $C_{T}$ method [15]. A t-test for independent samples (Analyze-it) was used to generate two-tailed $P$ statistics for each experiment. PCR primers were: Cezanne2 (exons 1112): RN2262: 5'-TGATTCACAAGCTCCCCTAGCT-3' (F), RN2263: 5'-GGAGTGGACCTGGGTTCATCT-3' (R); Chrna7 (exons 2-3): RN2357: 5'-GCCGCTCACCGTGTACTTCT-3' (F), RN2358: 5'-GGTGGTTAAAACTTGGTTCTTCTCA-3' (R); Luzp2 (exons 7-8): RN2264: 5'AAATCCAAGCCCAGCTGAAA-3' (F), RN2265: 5'-TGTTGGGCCTTAAATAACAAATCTT-3' (R); and Siglec-H (exons 1-2): RN2464: 5'-AGGATCTCTGTGCATGTGACAGA-3' (F), RN2465: 5'-AGGACGACCAAGCTCCAGTGT-3' (R).

\section{Regular RT-PCR}

Liver expression of Chrna7 was assessed in 2 TgPWS, 2 TgAS and $2 \mathrm{E}_{\mu} \mathrm{LMP} 2 \mathrm{~A}$ mice and WT littermates at P1. In addition, Chrna7 expression in spleen was determined in 2 TgAS vs. 2 WT mice at P1. Extraction and reverse transcription of total RNA was performed as for QRT-PCR experiments. Chrna7 primers (exons 1-3) were: RN2368: 5'-GGAGGCATCTGGCTGGCTCTG-3' (F), and RN2358 (R; see above). Siglec-H expression was performed using a Mouse Multiple Tissue cDNA Panel I (BD Bioscience Clontech) as well as WT brain at P1 and P4. Siglec-H primers (exons 1-2) were RN2511: 5'-GTGACAACGGTTCTTACT-3' (F) and RN2465 (R). For each gene analysis, RNApolII expression was used as a control: RN2318: 5'ACTCCTTCACTCACTGTCTTCCTGTT-3' (F), RN2319: 5'TCCTGATCTTCTGCCACCACTGT-3'(R). PCR conditions for Chrna7, Siglec-H, and RNApolII were initial denaturation at $94^{\circ} \mathrm{C}, 10 \mathrm{~min}$, followed by 32 cycles of denaturation at $94^{\circ} \mathrm{C}$ for $30 \mathrm{sec}$, annealing at $55^{\circ} \mathrm{C}, 30 \mathrm{sec}$, and extension at $72^{\circ} \mathrm{C}, 30 \mathrm{sec}$, with a final extension at $72^{\circ} \mathrm{C}$, 10 min, using Gold Taq DNA polymerase (ABI).

\section{NCCS analysis at the centromeric TgPWS/TgAS deletion breakpoint}

We identified non-coding conserved sequences (NCCS) by taking BAC genomic sequence, masking repetitive sequences with RepeatMasker [54], then using BLAST analysis of the non-redundant (NR) and whole genome shotgun (wgs) databases. From BAC RP24-426A19, we identified five NCCS elements (see Results), and PCR primers for NCCS1, NCCS3, NCCS4 and NCCS5 were designed and used to quantify the DNA amount in WT and TgPWS mice by Q-PCR. Brain and liver DNA were extracted using a phenol-chloroform method. $500 \mathrm{ng}$ of each DNA sample was used for Q-PCR and SYBR green was used as the fluorescent dye, with each Q-PCR reaction performed in triplicate. As an internal control for comparison of test DNA sequence, we used PCR for Gapdh intron 1: RN2518: 5'-GGCCGCCGCCATGT-3' (F) and RN2519: 5'-GGAAGGCCTAAGCAAGATTTCA-3' (R). In addition, primers from the Snurf-Snrpn promoter were used as a positive control for the deletion status in TgPWS DNA: RN2142: 5'-GCAAAAATGTGCGCATGTG-3' (F) and RN2143: 5'-CTCTCCTCTCTGCGCTAGTCTTG-3' (R). The $\mathrm{C}_{\mathrm{T}}$ method was used to process the data [15] and all the samples were normalized to the same WT sample. Primers for NCCS1 were: RN2515: 5'-AAATCATGAGCCAAGCCAAAA-3' (F) and RN2516: 5'-TGGCTTCCCTTATCACTTTCACA-3' (R); for NCCS3: RN2513: 5'TTGGAACATGCAGAACAATGAAT-3' (F) and RN2514: 5'AGGCTGCCAACCTGCAAA-3' (R); for NCCS4: RN2522: 5'-GCCACTAAATTGGATCCTTAGACATAT-3' (F) and RN2523: 5'-AAACCTGTTCCTACCCATGATAATCT-3' (R); and for NCCS5: RN2524: 5'-TCTCCCCCTAGGTCTTCTGTTTAA-3' (F) and RN2525: 5'-TGGCCAGTGATC ATGTACAGATC-3' (R).

\section{Fine mapping of the telomeric TgPWS/TgAS deletion breakpoint}

To fine map the telomeric TgPWS/TgAS breakpoint, total RNA from TgPWS brain tissue was reverse-transcribed using the Superscript First-Strand Synthesis System (Invitrogen) with a reverse primer from inside the transgene (Fig. 5A): OL-105: 5'-CGTGTGGCTTAC CTGCTGCCAATG-3'. cDNA was amplified by PCR using the RN2357 forward primer for Chrna7 (exon 2) and OL-105. The amplification conditions were initial denaturation at $94^{\circ} \mathrm{C}$ for $10 \mathrm{~min}$, followed by 37 cycles of denaturation at $94^{\circ} \mathrm{C}$ for $4 \mathrm{~min}$, annealing at $61^{\circ} \mathrm{C}$ for $1 \mathrm{~min}$, and extension at $72^{\circ} \mathrm{C}$ for $6 \mathrm{~min}$, with a final extension at $72^{\circ} \mathrm{C}$ for 10 min, using Platinum Pfx DNA polymerase (Invitrogen). PCR products were cloned into the pCR2.1-TA vector using a TOPO TA cloning kit (Invitrogen) and sequenced using standard techniques (University of Pennsylvania DNA sequencing Core facility).

\section{Orientation of the LMP2A transgene}

To determine the orientation of the LMP2A transgene array, three types of PCR amplifications were performed, first using forward and reverse primers located at the 3' and 5 ' ends of the transgene sequence, respectively (Fig. 5A): RN1316: 5'-GTTGTTTCCGCCATCGTACC-3' (R) and RN1317: 5'-TATGAGCTACTCTCTGACCC-3' (R). The other two reactions were performed using either forward or reverse primers alone. The amplification conditions were $95^{\circ} \mathrm{C}$ for $10 \mathrm{~min}$ followed by 32 cycles of denaturation at $95^{\circ} \mathrm{C}$ for $30 \mathrm{sec}$, annealing at $59^{\circ} \mathrm{C}$ for $30 \mathrm{sec}$, and extension at $72^{\circ} \mathrm{C}$ for $30 \mathrm{sec}$, with a final extension at $72^{\circ} \mathrm{C}$ for $10 \mathrm{~min}$, using Platinum Taq Gold polymerase (ABI).

\section{Authors' contributions}

MS prepared tissue samples, performed PCR, RT-PCR, QRT-PCR and QPCR studies, microarray and genome sequence analyses and drafted the manuscript. KCC carried out some RT-PCR experiments and performed the 
identification of the telomeric deletion breakpoint and DNA sequence analyses. ES performed FISH studies. JHC contributed to BAC contig assembly. TO performed transgene array analyses and designed these experiments. RL established the transgenic mouse lines and provided the $\mathrm{E}_{\mathrm{u}} \mathrm{LMP} 2 \mathrm{~A}$ mouse tissues. JMG designed, supervised and interpreted the FISH experiments. RDN designed the study, coordinated the project, performed genome sequence analysis, BAC contig assembly, participated in drafting and edited the manuscript.

\section{Acknowledgements}

We thank Brande Latney for technical assistance and Dr. Toni Portis for provision of tissues from $E_{\mu} L M P 2 A$ transgenic mice. This work was funded by grants to R.D.N. from the National Institutes of Health (HD3 49 I and ESI063I) and from the Foundation for Prader-Willi Research (FPWR). R.L. is supported by Public Health Service grants CA62234, CA73507, and CA93444 from the National Cancer Institute and DEI 3 I 27 from the National Institute of Dental and Craniofacial Research. M.S. was supported by a fellowship from the American Heart Association.

\section{References}

I. Nicholls RD, Knepper JL: Genome organization, function, and imprinting in Prader-Willi and Angelman syndromes. Annu Rev Genomics Hum Genet 200I, 2:153-175.

2. Cassidy SB, Schwartz S: Prader-Willi and Angelman syndromes. Disorders of genomic imprinting. Medicine (Baltimore) 1998, 77:|40-|5|.

3. Pärtsch CJ, Lammer C, Gillessen-Kaesbach G, Pankau R: Adult patients with Prader-Willi syndrome: clinical characteristics, life circumstances and growth hormone secretion. Growth Horm IGF Res 2000, 10:S8I-S85.

4. Amos-Landgraf JM, Ji Y, Gottlieb W, Depinet T, Wandstrat AE, Cassidy SB, Driscoll DJ, Rogan PK, Schwartz S, Nicholls RD: Chromosome breakage in the Prader-Willi and Angelman syndromes involves recombination between large, transcribed repeats at proximal and distal breakpoints. Am J Hum Genet 1999, 65:370-386.

5. Cattanach BM, Barr JA, Evans EP, Burtenshaw M, Beechey CV, Leff SE, Brannan $\mathrm{Cl}$, Copeland NG, Jenkins NA, Jones J: A candidate mouse model for Prader-Willi syndrome which shows an absence of Snrpn expression. Nat Genet 1992, 2:270-274.

6. Yang T, Adamson TE, Resnick JL, Leff S, Wevrick R, Francke U, Jenkins NA, Copeland NG, Brannan Cl: A mouse model for Prader-Willi syndrome imprinting-centre mutations. Nat Genet 1998, 19:25-31.

7. Gabriel JM, Merchant M, Ohta T, Ji Y, Caldwell RG, Ramsey MJ, Tucker JD, Longnecker R, Nicholls RD: A transgene insertion creating a heritable chromosome deletion mouse model of Prader-Willi and Angelman syndromes. Proc Natl Acad Sci USA 1999, 96:9258-9263.

8. Nicholls RD, Stefan M, Ji H, Qi Y, Frayo RS, Wharton RH, Dhar MS, Cummings DE, Friedman MI, Ahima RS: Mouse models for PraderWilli and Angelman syndromes offer insights into novel obesity mechanisms. Prog Obesity Res 2003:313-319.

9. Stefan M, Ji H, Simmons RA, Cummings DE, Ahima RS, Friedman MI, Nicholls RD: Hormonal and metabolic defects in a PraderWilli syndrome mouse model with neonatal failure to thrive. Endocrinology 2005, I 46:4377-4385.

10. Cattanach BM, Barr JA, Beechey CV, Martin J, Noebels J, Jones J: A candidate model for Angelman syndrome in the mouse. Mamm Genome 1997, 8:472-478.

II. Jiang YH, Armstrong D, Albrecht U, Atkins CM, Noebels JL, Eichele $G$, Sweatt JD, Beaudet AL: Mutation of the Angelman ubiquitin ligase in mice causes increased cytoplasmic p53 and deficits of contextual learning and long-term potentiation. Neuron 1998, 21:799-8II.

12. Miura K, Kishino T, Li E, Webber H, Dikkes P, Holmes GL, Wagstaff $\mathrm{J}$ : Neurobehavioral and electroencephalographic abnormali- ties in Ube3a maternal-deficient mice. Neurobiol Dis 2002, 9:149-159.

13. Chai JH, Locke DP, Ohta T, Greally JM, Nicholls RD: Retrotransposed genes such as Frat3 in the mouse Chromosome 7C Prader-Willi syndrome region acquire the imprinted status of their insertion site. Mamm Genome 200I, I2:8I3-82I.

14. Chai JH, Locke DP, Greally JM, Knoll JH, Ohta T, Dunai J, Yavor A, Eichler EE, Nicholls RD: Identification of four highly conserved genes between breakpoint hotspots BPI and BP2 of the Prader-Willi/Angelman syndromes deletion region that have undergone evolutionary transposition mediated by flanking duplicons. Am J Hum Genet 2003, 73:898-925.

15. Stefan M, Portis T, Longnecker R, Nicholls RD: A nonimprinted Prader-Willi Syndrome (PWS)-region gene regulates a different chromosomal domain in trans but the imprinted PWS loci do not alter genome-wide mRNA levels. Genomics 2005, 85:630-640.

16. Caldwell RG, Wilson JB, Anderson SJ, Longnecker R: Epstein-Barr virus LMP2A drives $B$ cell development and survival in the absence of normal B cell receptor signals. Immunity 1998, 9:405-4II.

17. Henegariu O, Heerema NA, Lowe Wright L, Bray-Ward P, Ward DC, Vance GH: Improvements in cytogenetic slide preparation: controlled chromosome spreading, chemical aging and gradual denaturing. Cytometry 2001, 43:101-109.

18. Greally JM, Gray TA, Gabriel JM, Song L, Zemel S, Nicholls RD: Conserved characteristics of heterochromatin-forming DNA at the I5q I I-q I3 imprinting center. Proc Nat Acad Sci USA I999, 96: I4430-I4435.

19. Mendell JT, Sharifi NA, Meyers JL, Martinez-Murillo F, Dietz HC: Nonsense surveillance regulates expression of diverse classes of mammalian transcripts and mutes genomic noise. Nat Genet 2004, 36: 1073-1078.

20. Angata T, Hingorani R, Varki NM, Varki A: Cloning and characterization of a novel mouse Siglec, mSiglec-F: differential evolution of the mouse and human (CD33) Siglec-3-related gene clusters. J Biol Chem 200I, 276:45 I28-45 I36.

21. Angata T, Margulies EH, Green ED, Varki A: Large-scale sequencing of the CD33-related Siglec gene cluster in five mammalian species reveals rapid evolution by multiple mechanisms. Proc Natl Acad Sci USA 2004, 10 I: 1 325 I- 13256.

22. Crocker PR: Siglecs: sialic-acid-binding immunoglobulin-like lectins in cell-cell interactions and signalling. Curr Opin Struct Biol 2002, 12:609-615.

23. Butler MG, Bittel DC, Kibiryeva N, Talebizadeh Z, Thompson T: Behavioral differences among subjects with Prader-Willi syndrome and type I or type II deletion and maternal disomy. Pediatrics 2004, I I 3:565-5673.

24. Varela MC, Kok F, Otto PA, Koiffmann CP: Phenotypic variability in Angelman syndrome: comparison among different deletion classes and between deletion and UPD subjects. Eur J Hum Genet 2004, I 2:987-992.

25. Wu M, Michaud EJ, Johnson DK: Cloning, functional study and comparative mapping of Luzp2 to mouse chromosome 7 and human chromosome IIpI3-IIpI4. Mamm Genome 2003, 14:323-334.

26. Orr-Urtreger A, Goldner FM, Saeki M, Lorenzo I, Goldberg L, De Biasi M, Dani JA, Patrick JW, Beaudet AL: Mice deficient in the alpha7 neuronal nicotinic acetylcholine receptor lack alphabungarotoxin binding sites and hippocampal fast nicotinic currents. J Neurosci 1997, 17:9165-9171.

27. Arredondo J, Nguyen VT, Chernyavsky AI, Bercovich D, OrrUrtreger A, Kummer W, Lips K, Vetter DE, Grando SA: Central role of alpha7 nicotinic receptor in differentiation of the stratified squamous epithelium. J Cell Biol 2002, I 59:325-336.

28. Morley BJ, Rodriguez-Sierra JF: A phenotype for the alpha 7 nicotinic acetylcholine receptor null mutant. Brain Res 2004, 1023:4|-47.

29. Bray C, Son JH, Kumar P, Meizel S: Mice deficient in CHRNA7, a subunit of the nicotinic acetylcholine receptor, produce sperm with impaired motility. Biol Reprod 2005, 73:807-814.

30. Stankiewicz P, Park SS, Inoue K, Lupski JR: The evolutionary chromosome translocation 4;19 in Gorilla gorilla is associated with microduplication of the chromosome fragment syntenic to sequences surrounding the human proximal CMTIA-REP. Genome Res 200I, I I:I205-1210. 
31. Bailey JA, Baertsch R, Kent WJ, Haussler D, Eichler EE: Hotspots of mammalian chromosomal evolution. Genome Biol 2004, 5:R23.

32. Mortlock DP, Portnoy ME, Chandler RL, Green ED: Comparative sequence analysis of the Gdf6 locus reveals a duplicon-mediated chromosomal rearrangement in rodents and rapidly diverging coding and regulatory sequences. Genomics 2004, 84:8I4-823.

33. MacDonald BT, Adamska M, Meisler MH: Hypomorphic expression of $D k k l$ in the doubleridge mouse: dose dependence and compensatory interactions with Lrp6. Development 2004, | 3 1:2543-2552.

34. Cunningham $D$, Xiao $Q$, Chatterjee $A$, Sulik K, Juriloff $D$, Elder $F$, Harrison W, Schuster G, Overbeek PA, Herman GE: Exma: an Xlinked insertional mutation that disrupts forebrain and eye development. Mamm Genome 2002, I3:|79-185.

35. Smiraglia DJ, Wu C, Ellsworth MK, Ratty AK, Chapman VM, Gross $\mathrm{KW}$ : Genetic characterization of the chromosomal rearrangements that accompany the transgene insertion in the chakragati mouse mutant. Genomics 1997, 45:572-579.

36. Kai M, Irie M, Okutsu $T$, Inoue K, Ogonuki N, Miki H, Yokoyama M, Migishima R, Muguruma K, Fujimura H, Kohda T, Ogura A, KanekoIshino T, Ishino F: The novel dominant mutation Dspd leads to a severe spermiogenesis defect in mice. Biol Reprod 2004, 70:1213-1221.

37. Morgan D, Turnpenny L, Goodship J, Dai W, Majumder K, Matthews L, Gardner A, Schuster G, Vien L, Harrison W, Elder FF, PenmanSplitt M, Overbeek P, Strachan T: Inversin, a novel gene in the vertebrate left-right axis pathway, is partially deleted in the inv mouse. Nat Genet 1998, 20:149-156. (Erratum in: 20, 312)

38. Qin Y, Kong LK, Poirier C, Truong C, Overbeek PA, Bishop CE: Long-range activation of Sox9 in Odd Sex (Ods) mice. Hum Mol Genet 2004, 13:12/3-12 18.

39. Bejerano G, Pheasant M, Makunin I, Stephen S, Kent WJ, Mattick JS, Haussler D: Ultraconserved elements in the human genome. Science 2004, 304: I 32I-I 325

40. Dermitzakis ET, Reymond A, Antonarakis SE: Conserved nongenic sequences - an unexpected feature of mammalian genomes. Nat Rev Genet 2005, 6: 15I-157.

4I. Loots GG, Locksley RM, Blankespoor CM, Wang ZE, Miller W, Rubin $E M$, Frazer $K A$ : Identification of a coordinate regulator of interleukins 4,13 , and 5 by cross-species sequence comparisons. Science 2000, 288: $136-140$.

42. Nobrega MA, Ovcharenko I, Afzal V, Rubin EM: Scanning human gene deserts for long-range enhancers. Science 2003, 302:413.

43. Nobrega MA, Pennacchio LA: Comparative genomic analysis as a tool for biological discovery. J Physiol 2004, 554:3I-39.

44. Gottgens B, Barton LM, Gilbert JG, Bench AJ, Sanchez MJ, Bahn S, Mistry S, Grafham D, McMurray A, Vaudin M, Amaya E, Bentley DR, Green AR, Sinclair AM: Analysis of vertebrate SCL loci identifies conserved enhancers. Nat Biotechnol 2000, 18:18I-186.

45. Henikoff S: Conspiracy of silence among repeated transgenes. Bioessays 1998, 20:532-535.

46. Freedman R, Coon $\mathrm{H}$, MylesWorsley M, OrrUrtreger A, Olincy A, Davis A, Polymeropoulos M, Holik J, Hopkins J, Hoff M, Rosenthal J, Waldo MC, Reimherr F, Wender P, Yaw J, Young DA, Breese CR, Adams C, Patterson D, Adler LE, Kruglyak L, Leonard S, Byerley W: Linkage of a neurophysiological deficit in schizophrenia to a chromosome 15 locus. Proc Natl Acad Sci USA 1997, 94:587-592.

47. Freedman R, Leonard S: Genetic linkage to schizophrenia at chromosome I5qI4. Am J Med Genet 200I, 105:655-657.

48. Perl O, Ilani T, Strous RD, Lapidus R, Fuchs S: The alpha7 nicotinic acetylcholine receptor in schizophrenia: decreased mRNA levels in peripheral blood lymphocytes. FASEB J 2003, I 7:1948-1950.

49. Leonard S, Gault J, Hopkins J, Logel J, Vianzon R, Short M, Drebing C Berger R, Venn D, Sirota P, Zerbe G, Olincy A, Ross RG, Adler LE, Freedman R: Association of promoter variants in the alpha7 nicotinic acetylcholine receptor subunit gene with an inhibitory deficit found in schizophrenia. Arch Gen Psychiatry 2002 59:1085-1096.

50. Gault J, Hopkins J, Berger R, Drebing C, Logel J, Walton C, Short M, Vianzon R, Olincy A, Ross RG, Adler LE, Freedman R, Leonard S: Comparison of polymorphisms in the alpha7 nicotinic receptor gene and its partial duplication in schizophrenic and control subjects. Am J Med Genet B Neuropsychiatr Genet 2003 , I 23:39-49.
5I. Houy E, Raux G, Thibaut F, Belmont A, Demily C, Allio G, Haouzir S, Fouldrin G, Petit M, Frebourg T, Campion D: The promoter-194 C polymorphism of the nicotinic alpha 7 receptor gene has a protective effect against the $\mathbf{P 5 0}$ sensory gating deficit. Mol Psychiatry 2004, 9:320-322.

52. Website title [http://www.ensembl.org/Mus musculus/]

53. Website title [http://www.ncbi.nlm.nih.gov/BLAST/]

54. Website title [http://www.repeatmasker.org/]
Publish with Biomed Central and every scientist can read your work free of charge

"BioMed Central will be the most significant development for disseminating the results of biomedical research in our lifetime. "

Sir Paul Nurse, Cancer Research UK

Your research papers will be:

- available free of charge to the entire biomedical community

- peer reviewed and published immediately upon acceptance

- cited in PubMed and archived on PubMed Central

- yours - you keep the copyright

Submit your manuscript here:

http://www.biomedcentral.com/info/publishing_adv.asp
BioMedcentral 